\title{
Stroke and Thromboembolism in Atrial Fibrillation
}

\author{
- Systematic Review of Stroke Risk Factors and \\ Risk Stratification Schema -
}

\author{
Ron Pisters, MD, PhD; Deirdre A. Lane, PhD; Francisco Marin, MD, PhD; \\ A. John Camm, MD; Gregory Y. H. Lip, MD
}

\begin{abstract}
We performed a systematic review of the available evidence on the relationship between the individual clinical, echocardiographic and laboratory characteristics of patients with atrial fibrillation (AF) and the risk of stroke. A systematic review was also performed of all published stroke risk stratification models, as well as the accuracy of their discriminative ability between risk strata. Third, we reviewed the literature on cost-effectiveness analyses with oral anticoagulation in AF. From the systematic review on stroke risk factors, a prior stroke or transient ischemic attack (15/16 studies positive, risk ratio [RR] 2.86), hypertension (11/20 studies positive, RR 2.27), aging (9/13 studies positive, RR 1.46 per decade increase), structural heart disease (9/13 studies positive, RR 2.0) and diabetes (9/14 studies positive, RR 1.62) were found to be good independent predictors of stroke. Supportive evidence was found for sex (8/22 studies positive, RR 1.67), vascular disease (6/17 studies positive, RR 2.61) and heart failure (7/18 studies positive, $R R$ 1.85). The various risk stratification schemes classified variable proportions as low, moderate and high risk, but the $\mathrm{CHA}_{2} \mathrm{DS}_{2}-\mathrm{VASc}$ score classified the smallest proportion of patients as 'low risk'. Anticoagulation with vitamin $\mathrm{K}$ antagonists and dabigatran is cost-effective in patients at high risk of stroke, but not in patients without any other stroke risk factor beside AF. Continued efforts are warranted to improve the antithrombotic management of AF patients to identify, and challenge, risk factors and refine risk stratification models in order to realize an individualized tailored, risk factor-based approach. (Circ J 2012; 76: 2289-2304)
\end{abstract}

Key Words: Atrial fibrillation; Risk factors; Stroke prevention

A trial fibrillation (AF) is the most common sustained arrhythmia ${ }^{1}$ and confers an independent risk of stroke and death. ${ }^{2}$ The individual AF patient's risk of stroke depends on the presence of other demographic, clinical, biochemical and echocardiographic risk factors. ${ }^{3,4}$ Oral anticoagulation (OAC) with vitamin $\mathrm{K}$ antagonists (VKAs) reduces the incidence of stroke by $64 \%$ and mortality by $26 \%$ compared to control or placebo, and is superior compared to the protection offered by antiplatelet agents. ${ }^{5}$ Recently, three novel OACs, 1 oral direct thrombin inhibitor (dabigatran) and 2 oral factor Xa inhibitors (rivaroxaban, apixaban), have been proven to match or even surpass the already impressive stroke risk reduction of VKAs. ${ }^{6-8}$

Transforming the data on stroke risk factors in AF patients into a user-friendly format that would be suitable for everyday clinical practice has been the rationale behind the development of the many risk stratification models (RSMs). RSMs initially aimed to classify patients into clinically relevant stroke risk categories (high-, intermediate- and low-risk) that could subsequently facilitate the choice of the most appropriate antithrombotic treatment. However, in clinical practice this translated into identifying the 'high-risk' AF patients who could be targeted for the 'inconvenient' VKAs, whereas all other AF patients could be treated with the less effective therapy, aspirin (ie, low- and intermediate-risk patients, or those who refuse VKAs) or no therapy. ${ }^{9-11}$

Over the past few years, a growing body of evidence has emerged to demonstrate that OAC, including VKAs, is beneficial even in patients with a 'moderate' stroke risk, including those with 1 stroke risk factor. ${ }^{12,13}$ Improvements in warfarin control, with a high percentage of time in therapeutic range (TTR), have contributed to lower bleeding rates (as well as low stroke rates). ${ }^{14}$

Thus, there has been a paradigm shift towards identification of 'truly low risk' individuals with AF who do not need any antithrombotic therapy, and those with $\geq 1$ stroke risk factors

Received August 17, 2012; accepted August 17, 2012; released online September 19, 2012

University of Birmingham Centre for Cardiovascular Sciences, City Hospital, Birmingham (R.P., D.A.L., G.Y.H.L.), UK; Department of Cardiology, Maastricht University Medical Centre, Maastricht (R.P.), the Netherlands; Department of Cardiology, Hospital Universitario Virgen de la Arrixaca, University of Murcia, Murcia (F.M.), Spain; and Division of Clinical Sciences, St. George's University of London, London (A.J.C.), UK

Mailing address: Gregory Y. H. Lip, Professor, MD, University of Birmingham Centre for Cardiovascular Sciences, City Hospital, Birmingham B18 7QH, UK. E-mail: g.y.h.lip@bham.ac.uk

ISSN-1346-9843 doi:10.1253/circj.CJ-12-1036

All rights are reserved to the Japanese Circulation Society. For permissions, please e-mail: cj@j-circ.or.jp 


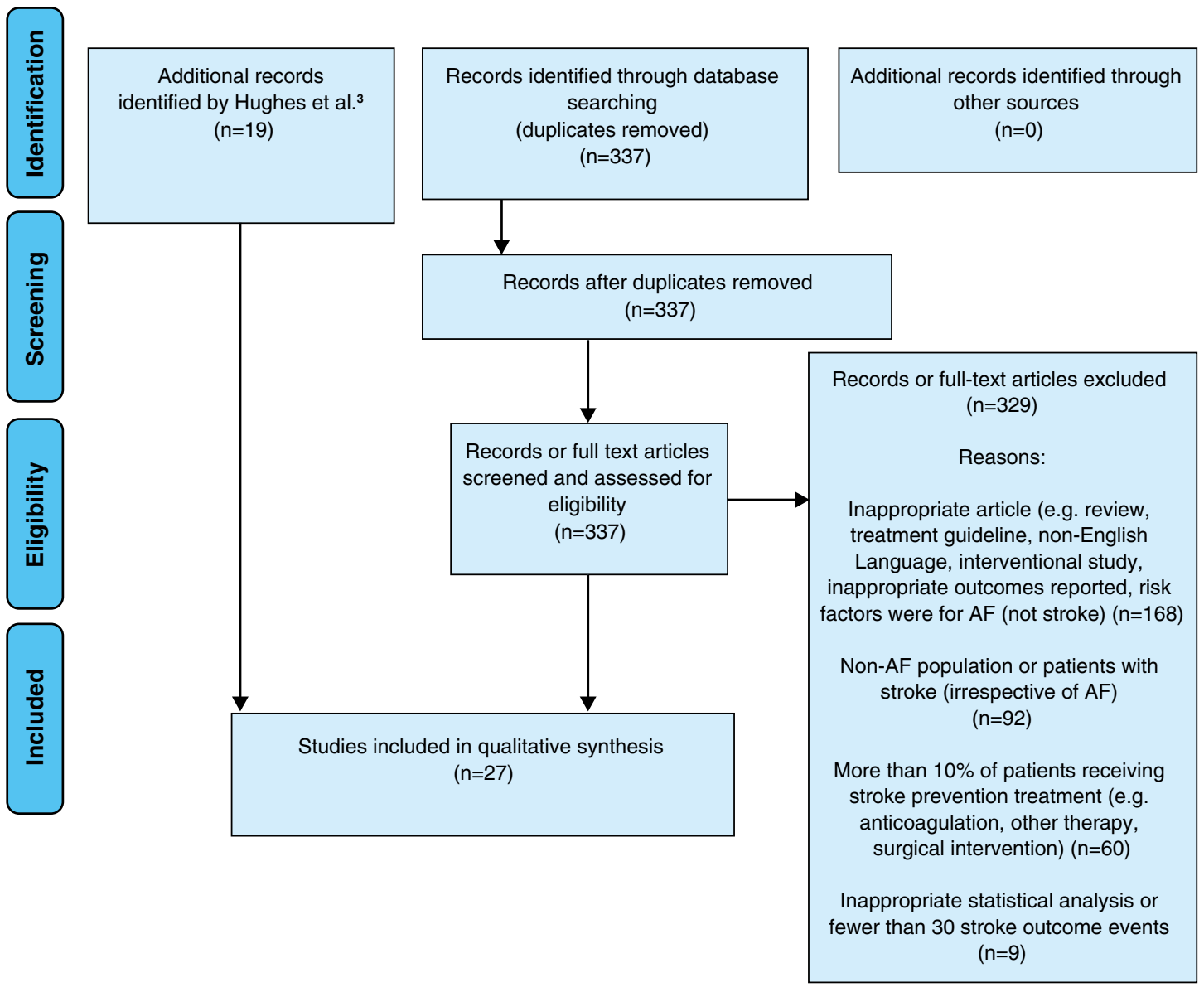

MeSH terms for database searches:

("atrial fibrillation"[MeSH Terms] OR ("atrial"[All Fields] AND "fibrillation"[All Fields]) OR "atrial fibrillation"[All Fields]) AND ("risk"[MeSH Terms] OR "risk"[All Fields]). The total number of hits (references) was 10,100.

Other searches pulled up similar references, for example:

(("patients"[MeSH Terms] OR "patients"[All Fields]) AND ("atrial fibrillation"[MeSH Terms] OR ("atrial"[All Fields] AND "fibrillation"[All Fields]) OR "atrial fibrillation"[All Fields])) AND (("risk"[MeSH Terms] OR "risk"[All Fields] OR "risk of"[All Fields]) AND ("stroke"[MeSH Terms] OR "stroke"[All Fields])). Total hits (references) = 3293.

(("patients"[MeSH Terms] OR "patients"[All Fields]) AND ("atrial fibrillation"[MeSH Terms] OR ("atrial"[All Fields] AND "fibrillation"[All Fields]) OR "atrial fibrillation"[All Fields])) AND ("risk"[MeSH Terms] OR "risk"[All Fields]) AND ("thromboembolism"[MeSH Terms] OR "thromboembolism"[All Fields]). Total hits (references) = 1251.

("cardiovascular system"[MeSH Terms] OR ("cardiovascular"[All Fields] AND "system"[All Fields]) OR "cardiovascular system"[All Fields] OR "cardiovascular"[All Fields]) AND (("risk"[MeSH Terms] OR "risk"[All Fields]) AND fibrillation[All Fields]).

Figure. PRISMA flow chart for identification and selection of studies reporting risk factors for stroke in patients with AF. AF, atrial fibrillation. 
who can be offered effective stroke prevention, which essentially is OAC-whether with well-controlled warfarin or one of the new OACs. Thus, a stroke risk factor (and not a category)based approach to thromboprophylaxis reflects current practice. ${ }^{15,16}$ In 2010, the European guidelines on the management of AF acknowledged the aforementioned evidence and subsequently adopted the revised, risk factor-based approach and emphasized that $\mathrm{OAC}$ is the recommended antithrombotic treatment for most patients with AF. ${ }^{17}$

The present analysis aimed to systematically review (a) the independent risk factors for stroke in AF patients, (b) the published RSMs and (c) the published cost-effectiveness data of (new) oral anticoagulant and antiplatelet agents for stroke prevention in $\mathrm{AF}$ patients.

\section{Methods}

Studies were selected for review based on the published abstract content (or full paper if no abstract was available) by 2 of the authors (R.P. and G.Y.H.L.). They evaluated all studies to see if they met the inclusion criteria to be considered for review. In case of disagreement, a third author was consulted. All decisions to exclude a study were unanimous. Details of the study selection criteria, outcome measures, systematic review methodology for the stroke risk factors/risk stratification methods and the cost-effectiveness review are provided in Appendix S1.

\section{Results}

\section{Stroke Risk Factors}

The systematic literature search yielded a total of 3,154 records leading to review for inclusion based on the full article in 432, of which 405 were excluded (see PRISMA flowchart in Figure). The remaining 27 studies were critically appraised. This systematic review allowed several studies that were derived from similar clinical trial populations to be included, because none of them used exactly the same populations or risk models.

Age Nine studies used a cut-off value (predominantly age $\geq 75$ years) ${ }^{15,18-25}$ and another 15 used increment per (half a) decade or age as a continuum for assessing its independent effect on stroke. , $19,24,26-35$ Of the 24 studies, 17 reported age to be an independent risk of stroke, ${ }^{4,19-23,25,27-29,33,34,36}$ whereas the other 7 studies did not (Table 1). ${ }^{15,18,24,30-32,40}$

A recent study by Hobbs et al studied the incremental risk per 5 years among 665 elderly patients (all aged $\geq 75$ years) and did not reveal age to be an independent stroke risk factor. ${ }^{30}$ However, the vast majority of the studies that looking at incremental risk per decade did find age to be an independent stroke risk factor. ${ }^{19,24,27-29,33,36}$ The evidence supporting age as an independent stroke risk factor also remains when it is considered for every decade of age increase, ${ }^{20-23}$ with only 2 negative results in a very small, older cohort ${ }^{18}$ and a mediumsize cohort of 1,577 patients across Europe. ${ }^{15}$

Sex Of the 21 studies considering biological sex as a risk factor for stroke (Table 2), 4,15,18,20,21,23-25,27-32,34-39 only 7 demonstrated that female sex was an independent risk factor for $\mathrm{s}$ troke. ${ }^{15,18,24,28,29,36,39}$ Another older study identified male sex as an independent risk factor for stroke in paroxysmal AF patients. ${ }^{20}$

Stroke, Transient Aschemic Attack (TIA) or Systemic Embolism All but one ${ }^{15}$ of 16 studies considering a history of stroke or TIA and 1 study considering thromboembolism found it to be a significant independent risk factor for recurrent stroke (Table 3). 4,21,24,25,28-30,33-36,38,40

Hypertension In total, 29 studies looked at whether hypertension was an independent stroke risk factor (Table 4). ${ }^{4,15,18,21-25,27-30,32-38,40-42}$ Only 9 studied a cut-off value of systolic blood pressure, mostly $>160 \mathrm{mmHg}$, as being predictive of stroke. ${ }^{24,25,28,29,36,41}$ This proved to be the case in 5 studies. ${ }^{24,25,28,29,36,41}$ The other 20 studies considered hypertension in general, including unspecified use of antihypertensive drugs and a history of hypertension, as a risk factor for stroke. $4,15,18,21-23,27-30,32-35,37,38,40,42$

In 13 of the 20 studies, ${ }^{4,21,22,27-29,35,37,40,42}$ including the largest of the 4 most recent studies in this regard, hypertension was an independent stroke risk factor, but this was not the case in the remaining 7 studies. ${ }^{15,18,23,30,32-34,38}$ Left ventricular hypertrophy, defined as a left ventricular mass $>110 \mathrm{~g} / \mathrm{m}^{2}$ in women and $>134 \mathrm{~g} / \mathrm{m}^{2}$ in men, was found to be a significant independent risk factor for stroke in 2 studies. ${ }^{38,41}$

Chronic Kidney Disease Only 2 studies examined the relationship between chronic kidney disease and stroke, with 1 not demonstrating an independent association with the risk of stroke ${ }^{21}$ and the other study reported a correlation between decreasing glomerular filtration rate and stroke risk in conjunction with proteinuria as an independent stroke risk factor. ${ }^{43}$

Structural Heart Disease Structural heart disease in general was recently found not to be predictive of stroke in a UK primary care study considering valvular and congenital cardiac disease, ${ }^{23}$ whereas an older, single study did show the predictive ability of structural heart disease ${ }^{18}$ (Table 5A). Valvular heart disease in general, and mitral stenosis specifically, were found to be independent risk factors for stroke in 2 studies, ${ }^{25,38}$ but not in another, ${ }^{30}$ and there was no relationship between annular calcification and stroke. ${ }^{37}$

A meta-analysis of 3 clinical trials did not demonstrate that any degree of mitral valve prolapse or regurgitation was an independent predictor of stroke or thromboembolism. ${ }^{34} \mathrm{~A}$ dilated left atrium ${ }^{37}$ and increasing left atrial size per body surface area $^{26}$ were found to be independent stroke risk factors.

Vascular Disease Ischemic heart disease was not an independent stroke risk factor in 3 studies. ${ }^{21,27} \mathrm{~A}$ similar conclusion was drawn from 2 other studies in respect to angina., 40 History of myocardial infarction was considered in 6 studies,

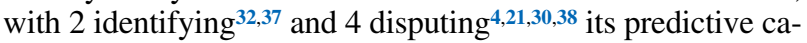
pability regarding stroke risk.

More recently, peripheral artery disease alone has been examined as a stroke risk factor in 2 cohorts, both identifying it as an independent stroke risk factor. ${ }^{21,23}$ Two other studies investigated vascular disease as a combination of peripheral artery and/or coronary artery disease, ${ }^{15,44}$ with 1 reporting an independent relationship, ${ }^{44}$ whereas the other ${ }^{15}$ did not identify this combination of vascular disease to be an independent stroke risk factor.

Congestive Heart Failure (CHF) A history or current episode of $\mathrm{CHF}$ was considered as a risk factor for stroke in 12 studies. $4,15,21,25,27,28,30,33-35,40$ Nine studies found that CHF was not an independent risk factor for stroke. , $15,28,30,33-35,40$ The remaining 3 , including the largest cohort of the most recent studies, reported that CHF independently predicted stroke. ${ }^{21,25,34}$

Left ventricular dysfunction (LVD), being a mix of definitions of recent $\mathrm{CHF}$ or more quantitative measures (left ventricular fractional shortening $\leq 25 \%$ or left ventricular ejection fraction $\leq 50$ or $40 \%$ ), was considered in 8 studies, ${ }^{15,26,28,33,35,38,41}$ with only half reporting that LVD was a significant independent stroke risk factor. ${ }^{26,34,38,41}$

Other Risk Factors Table 5B provides an overview of the studies which considered other (predisposing factors of) car- 
Table 1. Summary of Results for Increasing Age as a Risk Factor for Stroke in Terms of Whether or Not the Study Reported Increasing Age as a Significant Risk Factor

\begin{tabular}{|c|c|c|c|c|c|c|}
\hline Study & $\mathbf{n}$ & Age risk factor & $\begin{array}{l}\text { Stroke rate } \\
\text { test }\end{array}$ & $\begin{array}{l}\text { Stroke rate } \\
\text { ref. }\end{array}$ & RR/OR/HR (95\% Cl) & $P$ value \\
\hline Cabin HS, $1990^{18}$ & 272 & $\begin{array}{l}\text { Age }>70 \text { vs. } \leq 70 \\
\text { years }\end{array}$ & $13 \%$ & $7 \%$ & NA (bivariate analysis) & NS \\
\hline Petersen $\mathrm{P}, 1990^{32}$ & 336 & $\begin{array}{l}\text { Correlation with } \\
\text { increasing age }\end{array}$ & NA & NA & LR Enter 0.01 & NS \\
\hline Moulton AW, $1991^{22}$ & 265 & $\begin{array}{l}\text { Age }>75 \text { vs. } \leq 75 \\
\text { years }\end{array}$ & NA & NA & OR $1.72(1.04-2.84)$ & $<0.05$ \\
\hline SPAF Investigators, $1992^{40}$ & 568 & $\begin{array}{l}\text { Incremental risk per } \\
\text { decade }\end{array}$ & NA & NA & RR $1.2(0.9-1.6)$ & NS \\
\hline $\begin{array}{l}\text { Laupacis A, } 1994^{4} \text { (AF Invest. } \\
\text { 1994) }\end{array}$ & 1,593 & $\begin{array}{l}\text { Incremental risk per } \\
\text { decade }\end{array}$ & NA & NA & RR 1.4 & $<0.05$ \\
\hline \multirow[t]{3}{*}{ Van Latum JC, $1995^{24}$} & 375 & $\begin{array}{l}\geq 60-69 \text { vs. }<60 \\
\text { years }\end{array}$ & NA & NA & HR $0.8(0.3-1.9)$ & NS \\
\hline & & $\begin{array}{l}\geq 70-79 \text { vs. }<60 \\
\text { years }\end{array}$ & NA & NA & HR $0.9(0.4-2.1)$ & NS \\
\hline & & $\geq 80$ vs. $<60$ years & NA & NA & HR $0.6(0.2-1.6)$ & NS \\
\hline $\begin{array}{l}\text { Ezekowitz M, } 1998^{34} \text { (using } \\
\text { clinical factors alone) }\end{array}$ & 1,066 & $\begin{array}{l}\text { Incremental risk per } \\
\text { decade }\end{array}$ & NA & NA & RR $1.5(1.1-1.9)$ & 0.008 \\
\hline $\begin{array}{l}\text { Ezekowitz M, } 1998^{34} \text { (using } \\
\text { clinical and echo- cardio- } \\
\text { graphic features) }\end{array}$ & 1,010 & $\begin{array}{l}\text { Incremental risk per } \\
\text { decade }\end{array}$ & NA & NA & RR $1.5(1.1-2.0)$ & 0.006 \\
\hline Nakagami H, $1998^{31}$ & 290 & $\begin{array}{l}\text { Incremental risk per } \\
\text { decade }\end{array}$ & NA & NA & OR $1.33(1.04-1.71)$ & NS \\
\hline SPAF III, $1998^{27}$ & 892 & $\begin{array}{l}\text { Incremental risk per } \\
\text { decade }\end{array}$ & NA & NA & RR $1.7(1.1-2.6)$ & 0.01 \\
\hline Stollberger C, $1998^{35}$ & 409 & $\begin{array}{l}\text { Correlation with } \\
\text { increasing age }\end{array}$ & NA & NA & RR $1.1(1.0-1.11)$ & $<0.001$ \\
\hline Hart RG, $1999^{28}$ & 2,012 & $\begin{array}{l}\text { Incremental risk per } \\
\text { decade }\end{array}$ & NA & NA & RR 1.8 & $<0.001$ \\
\hline $\begin{array}{l}\text { Hart RG, } 2000^{29} \text { (paroxysmal } \\
\text { AF) }\end{array}$ & 460 & $\begin{array}{l}\text { Incremental risk per } \\
\text { decade }\end{array}$ & NA & NA & RR 2.1 & $<0.001$ \\
\hline $\begin{array}{l}\text { Hart RG, } 2000^{29} \text { (persistent } \\
\text { AF) }\end{array}$ & 1,552 & $\begin{array}{l}\text { Incremental risk per } \\
\text { decade }\end{array}$ & NA & NA & RR 1.7 & $<0.001$ \\
\hline Inoue $\mathrm{H}, 2000^{20}$ & 740 & Age $>65$ years & NA & NA & RR 3.33 (1.92-5.81) & 0.0001 \\
\hline \multirow[t]{2}{*}{ Wang TJ, $2003^{36}$} & 705 & $\begin{array}{l}\text { Incremental risk per } \\
\text { decade }\end{array}$ & NA & NA & $\begin{array}{l}\text { HR } 1.32(1.02-1.76) \\
\text { (for stroke) }\end{array}$ & $<0.05$ \\
\hline & & $\begin{array}{l}\text { Incremental risk per } \\
\text { decade }\end{array}$ & NA & NA & $\begin{array}{l}\text { HR } 2.13 \text { (1.86-2.44) } \\
\text { (for stroke or death) }\end{array}$ & $<0.05$ \\
\hline Stollberger C, $2004^{33}$ & 409 & $\begin{array}{l}\text { Correlation with } \\
\text { increasing age }\end{array}$ & NA & NA & RR 1.05 (1.02-1.09) & 0.0006 \\
\hline Frost L, $2007^{19}$ & 141,493 & $\begin{array}{l}\text { Age } 70-74 \text { vs. } 40-44 \\
\text { years (for men) }\end{array}$ & NA & NA & HR 6.31 (4.78-8.33) & $<0.05$ \\
\hline Ruigómez A, $2009^{23}$ & 831 & $\begin{array}{l}\text { Age }>80 \text { vs. } 40-59 \\
\text { years }\end{array}$ & NA & NA & RR 5.8 (1.9-17.6) & $<0.05$ \\
\hline Lip GYH, $2010^{15}$ & 1,577 & $\begin{array}{l}\text { Age }>75 \text { vs. } \leq 75 \\
\text { years }\end{array}$ & $3.6 \%$ & $1.8 \%$ & OR 1.46 (0.63-3.65) & NS \\
\hline Hobbs FD, $2011^{30}$ & 665 & $\begin{array}{l}\text { Incremental risk per } \\
5 \text { years }\end{array}$ & NA & NA & HR 1.26 (0.93-1.72) & NS \\
\hline Lin LY, $2011^{21}$ & 7,920 & $\begin{array}{l}\text { Age } \geq 75 \text { vs. } 20-64 \\
\text { years }\end{array}$ & NA & NA & OR $1.652(1.313-2.080)$ & $<0.001$ \\
\hline \multirow[t]{4}{*}{ Van Staa TP, $2011^{25}$} & 79,844 & $\begin{array}{l}\text { Age } 50-59 \text { vs. } 60-69 \\
\text { years }\end{array}$ & NA & NA & RR $0.44(0.28-0.69)$ & $<0.05$ \\
\hline & & $\begin{array}{l}\text { Age }<50 \text { vs. } 60-69 \\
\text { years }\end{array}$ & NA & NA & RR $0.14(0.06-0.34)$ & $<0.05$ \\
\hline & & $\begin{array}{l}\text { Age } \geq 80 \text { vs. } 60-69 \\
\text { years }\end{array}$ & NA & NA & RR 2.22 (1.78-2.76) & $<0.05$ \\
\hline & & $\begin{array}{l}\text { Age } 70-79 \text { vs. } 60-69 \\
\text { years }\end{array}$ & NA & NA & RR 1.42 (1.12-1.78) & $<0.05$ \\
\hline
\end{tabular}

$\mathrm{AF}$, arial fibrillation; $\mathrm{Cl}$, confidence interval; $\mathrm{HR}$, hazard ratio; LR, likelihood ratio; $\mathrm{n}$, sample size; NA, not available; NS, not significant (P>0.05); $\mathrm{OR}$, odds ratio; $\mathrm{RR}$, risk ratio. 


\begin{tabular}{|c|c|c|c|c|c|c|}
\hline Study & $\mathbf{n}$ & $\begin{array}{c}\text { Sex } \\
\text { comparison }\end{array}$ & $\begin{array}{l}\text { Stroke rate } \\
\text { test }\end{array}$ & $\begin{array}{l}\text { Stroke rate } \\
\text { ref. }\end{array}$ & RR/OR/HR (95\% Cl) & $P$ value \\
\hline Aronow WS, $1989^{37}$ & 110 & F vs. M & NA & NA & OR 0.85 & NS \\
\hline \multirow[t]{2}{*}{ Cabin HS, $1990^{18}$} & 272 & F vs. M & $14 \%$ & $6 \%$ & NA (bivariate analysis) & 0.023 \\
\hline & & & & & CRC 1.0 & 0.014 \\
\hline Petersen P, $1990^{32}$ & 336 & F vs. M & NA & NA & LR Enter 0.48 & NS \\
\hline Laupacis A, 1994 (AF Investig. 1994) & 1,593 & F vs. M & NA & NA & NA & NS \\
\hline Van Latum JC, $1995^{24}$ & 375 & Fvs. M & NA & NA & HR $1.5(1.0-2.4)$ & NR \\
\hline $\begin{array}{l}\text { Ezekowitz M, } 1998^{34} \text { (using clinical } \\
\text { factors alone) }\end{array}$ & 1,066 & F vs. M & NA & NA & NA & NS \\
\hline Aronow WS, $1998^{38}$ & 312 & M vs. F & NA & NA & RR 0.98 (0.67-1.43) & NS \\
\hline Nakagami H, $1998^{31}$ & 290 & F vs. M & NA & NA & OR $0.98(0.55-1.72)$ & NS \\
\hline SPAF III, $1998^{27}$ & 892 & F vs. M & NA & NA & NA & NS \\
\hline Stollberger CM, $1998^{35}$ & 409 & F vs. M & NA & NA & NS & NS \\
\hline Hart RG, $1999^{28}$ & 2,012 & F vs. M & NA & NA & RR 1.6 & 0.01 \\
\hline Hart RG, $2000^{29}$ (paroxysmal AF) & 460 & Fvs. M & NA & NA & NA & NS \\
\hline Hart RG, $2000^{29}$ (persistent AF) & 1,552 & F vs. M & NA & NA & RR 1.8 & 0.004 \\
\hline Inoue $\mathrm{H}, 2000^{20}$ & 740 & M vs. F & NA & NA & OR 2.00 (1.07-3.72) & 0.0291 \\
\hline Wang TJ, $2003^{36}$ & 705 & F vs. M & NA & NA & $1.92(1.20-3.07)$ & $<0.05$ \\
\hline Fang $\mathrm{M}, 2005^{39}$ & 13,559 & F vs. M & $3.5 \%$ & $1.8 \%$ & RR $1.6(1.3-1.9)$ & $<0.05$ \\
\hline Ruigomez A, $2009^{23}$ & 831 & M vs. F & NA & NA & RR $1.0(0.6-1.7)$ & NS \\
\hline Lip GYH, $2010^{15}$ & 1,084 & F vs. M & $3.6 \%$ & $1.4 \%$ & OR 2.53 (1.08-5.92) & 0.03 \\
\hline Hobbs FD, $2011^{30}$ & 665 & F vs. M & NA & NA & HR $0.99(0.57-1.70)$ & NS \\
\hline Lin LY, $2011^{21}$ & 7,920 & F vs. M & NA & NA & OR $0.942(0.787-1.127)$ & NS \\
\hline Van Staa TP, $2011^{25}$ & 79,844 & M vs. F & NA & NA & RR 0.95 (0.84-1.06) & NS \\
\hline
\end{tabular}

CRC, Cox regression coefficient; F, female; M, male; NR, not reported. Other abbreviations as in Table 1.

\begin{tabular}{|c|c|c|c|c|c|}
\hline Study & $\mathbf{n}$ & $\begin{array}{c}\text { Stroke } \\
\text { rate test }\end{array}$ & $\begin{array}{c}\text { Stroke } \\
\text { rate ref. }\end{array}$ & $\begin{array}{c}\text { RR/OR/HR } \\
(95 \% \mathrm{Cl})\end{array}$ & $P$ value \\
\hline SPAF I, $1992^{40}$ & 568 & NA & NA & RR $2.1(1.0-4.2)$ & 0.04 \\
\hline Laupacis A, $1994^{4}$ (AF Investig. 1994) & 1,593 & NA & NA & $\mathrm{RR} 2.5$ & $<0.05$ \\
\hline Van Latum JC, $1995^{24}$ & 375 & NA & NA & HR $1.6(1.0-2.6)$ & $<0.05$ \\
\hline $\begin{array}{l}\text { Ezekowitz M, } 1998^{34} \text { (using clinical factors } \\
\text { alone) }\end{array}$ & 1,066 & NA & NA & RR $3.4(1.8-6.6)$ & $<0.001$ \\
\hline $\begin{array}{l}\text { Ezekowitz M, } 1998^{34} \text { (using clinical and echo- } \\
\text { cardiographic features) }\end{array}$ & 1,010 & NA & NA & RR $3.5(1.8-6.7)$ & $<0.001$ \\
\hline Aronow WS, $1998^{38}$ & 312 & NA & NA & RR $1.574(1.12-2.21)$ & 0.009 \\
\hline Stollberger C, $1998^{35}$ & 409 & NA & NA & RR 3.7 (1.5-7.5) & 0.002 \\
\hline Hart RG, $1999^{28}$ & 2,012 & NA & NA & RR 2.9 & $<0.001$ \\
\hline Hart RG, $2000^{29}$ (paroxysmal AF) & 460 & NA & NA & RR 4.1 & 0.01 \\
\hline Hart RG, $2000^{29}$ (persistent AF) & 1,552 & NA & NA & RR 2.7 & $<0.001$ \\
\hline Wang TJ, $2003^{36}$ & 705 & NA & NA & HR 1.88 (1.09-3.26) & $<0.05$ \\
\hline Stollberger C, 2004 ${ }^{33}$ & 409 & NA & NA & RR 2.14 (1.02-4.51) & 0.0454 \\
\hline Hobbs FD, $2011^{30}$ & 665 & NA & NA & HR 2.59 (1.36-4.90) & 0.003 \\
\hline Lin LY, $2011^{21}$ & 7,920 & NA & NA & OR $2.752(2.06-3.68)$ & $<0.001$ \\
\hline Lip GYH, $2010^{15}$ & 1,577 & $5.9 \%$ & $2.0 \%$ & OR 2.22 (0.78-6.35) & NS \\
\hline Van Staa TP, $2011^{25}$ & 79,844 & NA & NA & RR 2.86 (2.53-3.22) & $<0.05$ \\
\hline
\end{tabular}

TIA, transient ischemic attack. Other abbreviations as in Tables 1,2.

diovascular disease as risk factors for stroke. Of these, 2 studies failed to find that being a smoker was an independent risk factor for stroke., ${ }^{4,23}$ Obesity was considered in 3 studies and only to be found predictive of stroke in one. One study considered alcohol abuse and hyperlipidaemia, but neither was found to be independent stroke risk factor. ${ }^{23}$
Nine studies found diabetes mellitus to be an independent risk factor for stroke. ${ }^{4,21,25,28,30,34,36}$ Five other studies, 1 in a general population, ${ }^{15} 1$ in patients with atrial flutter, ${ }^{42} 1$ in an elderly cohort ${ }^{38}$ and 2 in low-risk AF patients, ${ }^{27,32}$ failed to find that diabetes mellitus was an independent stroke risk factor.

Hormone replacement therapy was found to independently 
Table 4. Summary of Results for Htn as a Risk Factor for Stroke in Terms of Whether or Not the Study Reported Htn as a Significant Risk Factor

\begin{tabular}{|c|c|c|c|c|}
\hline Study & $\mathbf{n}$ & $\begin{array}{l}\text { Stroke } \\
\text { rate test }\end{array}$ & $\begin{array}{c}\text { Stroke } \\
\text { rate ref. }\end{array}$ & $\begin{array}{c}\text { RR/OR/HR } \\
(95 \% \mathrm{Cl})\end{array}$ \\
\hline
\end{tabular}

(A) SBP $>160 \mathrm{mmHg}$ as a risk factor for stroke in terms of whether or not the study reported SBP $>160 \mathrm{mmHg}$ as a significant risk factors

\begin{tabular}{|c|c|c|c|c|c|}
\hline SPAF study, $1995^{41}$ & 854 & NA & NA & RR $2.2(1.3-3.6)$ & 0.004 \\
\hline Van Latum JC, $1995^{24}$ & 375 & NA & NA & HR $1.7(1.0-2.9)$ & NR \\
\hline Hart RG, $1999^{28}$ & 2,012 & NA & NA & RR 2.3 & $<0.001$ \\
\hline Hart RG, $2000^{29}$ (paroxysmal AF) & 460 & NA & NA & NA & NS \\
\hline Hart RG, $2000^{29}$ (persistent AF) & 1,552 & NA & NA & RR 2.8 & $<0.001$ \\
\hline \multirow[t]{2}{*}{ Wang TJ, $2003^{36}$ (per $10 \mathrm{mmHg}$ increase) } & 705 & NA & NA & $\begin{array}{l}\text { HR } 1.06(0.97-1.17) \\
\text { (for stroke) }\end{array}$ & NS \\
\hline & & NA & NA & $\begin{array}{l}\text { HR } 1.06(1.02-1.11) \\
\text { (for stroke or death) }\end{array}$ & $<0.05$ \\
\hline $\begin{array}{l}\text { van Staa TP, } 2011^{25} \text { (140-159 vs. } \\
<120 \mathrm{mmHg} \text { ) }\end{array}$ & 79,844 & NA & NA & RR $2.74(1.21-6.19)$ & $<0.05$ \\
\hline $\begin{array}{l}\text { van Staa TP, } 2011^{25} \text { (160-179 vs. } \\
<120 \mathrm{mmHg})\end{array}$ & 79,844 & NA & NA & RR $1.49(0.55-4.00)$ & NS \\
\hline van Staa TP, $2011^{25}$ ( $\geq 180$ vs. $<120 \mathrm{mmHg}$ ) & 79,844 & NA & NA & RR $4.28(1.25-14.64)$ & $<0.05$ \\
\hline
\end{tabular}

(B) Presence of $\mathrm{Htn}$ (including a history of $\mathrm{Htn}$ ) as a risk factor for stroke in terms of whether or not the study reported $\mathrm{Htn}$ as a significant risk factor

Cabin HS, $1990^{18}$

Petersen P, $1990^{32}$

$$
272
$$

Moulton AW, $1991^{22}$

SPAF Investigators, $1992^{40}$

Laupacis A, 19944 (AF Investig. 1994)

Ezekowitz M, $1998^{27}$ (using clinical factors alone)

Ezekowitz M, $1998^{34}$ (using clinical and echocardiographic features)

Aronow WS, $1998^{38}$

Seidl K, $1998^{42}$

SPAF III, $1998^{27}$

Stollberger C, $1998^{35}$

Hart RG, $1999^{28}$

Hart RG, $2000^{29}$ (paroxysmal AF)

Hart RG, 200029 (persistent AF)

Stollberger C, $2004^{33}$

Ruigomez A, $2009^{23}$

Lip GYH, $2010^{15}$

Hobbs FD, $2011^{30}$

Lin LY, $2011^{21}$

$\begin{array}{lr}312 \\ 191 \\ 892 \\ 409 \\ & 2,012 \\ & 460 \\ & 1,552 \\ 409 \\ 831 \\ 1,084 \\ 665 \\ 7,920\end{array}$

$\begin{array}{ll}\text { NA } & \text { NA } \\ 12 \% & 8 \% \\ \text { NA } & \text { NA } \\ \text { NA } & \text { NA } \\ \text { NA } & \text { NA } \\ \text { NA } & \text { NA } \\ \text { NA } & \text { NA } \\ \text { NA } & \end{array}$

NA
$8 \%$
NA
NA
NA
NA
NA

$\begin{array}{cr}\text { OR } 12.51 & <0.01 \\ \text { NA (bivariate analysis) } & \text { NS } \\ \text { LR Enter 0.8 } & \text { NS } \\ \text { OR 1.89 }(1.15-3.10) & <0.05 \\ \text { RR } 2.2(1.1-4.3) & 0.02 \\ \text { RR 1.6 } & <0.05 \\ \text { RR 1.5 }(0.9-2.5) & 0.11 \\ & \\ \text { RR 1.5 }(0.9-2.5) & 0.13\end{array}$

NA NA

NA NA

$3.6 \%$ per year $1.1 \%$ per year

NA NA

NA NA

NA NA

NA NA

NA NA

NA NA

$2.6 \% \quad 1.7 \%$

NA NA

NA NA

$\begin{array}{cc}\text { RR 1.5 (0.9-2.5) } & 0.13 \\ \text { RR 1.254 (0.847-1.855) } & \text { NS } \\ \text { OR 6.5 (1.5-45) } & <0.05 \\ \text { RR 3.3 (1.7-6.9) } & 0.001 \\ \text { RR 3.6 (1.8-8.4) } & 0.001 \\ \text { RR 2.0 } & <0.001 \\ \text { RR 3.4 } & 0.003 \\ \text { RR 1.8 } & 0.008 \\ \text { NA } & \text { NS } \\ \text { RR 0.6 (0.3-1.1) } & \text { NS } \\ \text { OR 1.01 (0.38-2.66) } & \text { NS } \\ \text { HR 1.10 (0.62-1.95) } & \text { NS } \\ \text { OR 2.656 }(2.140-3.296) & <0.001\end{array}$

Htn, hypertension. Other abbreviations as in Tables 1,2.

predict the risk of stroke in $\mathrm{AF}$ patients in 1 study, ${ }^{28}$ but not another. ${ }^{39}$

One population-based prospective study investigating whether or not the blood coagulation markers, von Willebrand factor, soluble P-selectin and fibrinogen were independent predictors of stroke and cardiovascular mortality in AF patients failed to demonstrate an independent risk for stroke associated with any of these parameters. ${ }^{45}$ Another study identified Creactive protein as a predictor risk of stroke. ${ }^{25}$

AF Type Paroxysmal vs. non-paroxysmal clinical type of $\mathrm{AF}$ was not found to independently predict stroke in any of the 3 studies that considered it. ${ }^{18,33,46}$ In only 1 study was a longer history of AF found to be an independent stroke risk factor. ${ }^{24}$

\section{Stroke Risk Stratification Models}

The same 3,154 abstracts were used for the systematic review of RSMs. The abstract and full text information led to the exclusion of 3,135 studies and inclusion of 19 studies. A general overview of all included studies in the systematic review of RSMs is shown in Table $6 \mathbf{A}$.

First, the incorporation of independent risk factors of stroke derived from the systematic review of stroke risk factors into the RSMs was checked (Table 6B). The risk factors used in all RSMs were acknowledged to be independent risk factors of stroke by at least one of the studies from the systematic review, except for $2 .{ }^{47,48}$ These RSMs were both based on expert consensus. Although some definition of hypertension could be found in all RSMs, this was not the case for the strongest independent risk factor of stroke: prior stroke or thromboem- 
Table 5. Summary of Results for (A) the Presence of SHD as a Risk Factor for Stroke in Terms of Whether or Not the Study Reported SHD as a Significant Risk Factor and (B) Other Cardiovascular Conditions and Risk Factors in Terms of Whether or Not the Study Reported the Respective Condition

(A)

\begin{tabular}{|c|c|}
\hline Study & $\mathbf{n}$ \\
\hline SPAF III, $1998^{27}$ & \\
\hline Stollberger CM, $1998^{35}$ & \\
\hline Aronow WS, $1989^{37}$ & \\
\hline Petersen P, $1990^{32}$ & \\
\hline Laupacis A, 19944 (AF Investig. 1994) & 1,59 \\
\hline Aronow WS, $1998^{38}$ & \\
\hline Hobbs FDR, $2011^{30}$ & 66 \\
\hline Lin LY, $2011^{21}$ & 7,9 \\
\hline Lin LY, $2011^{21}$ & 7,92 \\
\hline Cabin HS, $1990^{18}$ & \\
\hline
\end{tabular}
831

Laupacis A, $1994^{4}$ (AF Investig. 1994)

Hobbs FD, 201130

1,593

SPAF Investig., $1992^{26}$

Laupacis A, $1994^{4}$ (AF Investigators

1994

Ezekowitz M, $1998^{34}$ (using clinical

factors alone)

Ezekowitz M, 1998 ${ }^{34}$ (AF Investig. 1998) $\quad 1,010$

(using clinical and echocardiographic

features)

SPAF III, $1998^{27}$

Hart RG, $1999^{28}$

Lip GYH, $2010^{15}$

Hobbs FD, $2011^{30}$

Lin LY, $2011^{21}$

Van Staa TP, $2011^{25}$

SPAF Investig., $1992^{26}$

SPAF Study, $1995^{41}$

AFI Echo, 1998 ${ }^{34}$ (AF Investig. 1998)

(Using clinical and echocardiographic features)

Aronow WS, $1998^{38}$

Stollberger CM, $1998^{35}$

Stollberger CM, 2004 33

Stollberger CM, $1998^{35}$

Stollberger CM, 2004 33

892

2,012

1,577

665

7,920

79,844

568

854

1,010

Hart RG, $1999^{28}$

Lip GYH, 201015

Aronow WS, $1989^{37}$

Aronow WS, $1998^{38}$

AFI Echo, 1998 (AF Investig. 1998)

(using clinical and echocardiographic features)

AFI Echo, 1998 (AF Investig. 1998)

(using clinical and echocardiographic features)

Aronow WS, $1998^{38}$

Aronow WS, $1998^{38}$

Nakagami, $1998^{31}$

SHD definition
Ischemic heart disease
Ischemic heart disease
$\mathrm{MI}$

Stroke Stroke

rate test rate ref.

NA NA

NA NA

NA NA

$28 \% / 2$ y $\quad 11 \% / 2$ y

MI

$\mathrm{Ml}$

MI

Coronary artery disease

MI

Structural heart disease

Structural heart disease

Structural heart disease

(other cardiac disease)

Angina

Angina

CHF

CHF

CHF

CHF

CHF

CHF

$\mathrm{CHF}$

$\mathrm{CHF}$

$\mathrm{CHF}$

CHF

LV dysfunction

LV dysfunction

LV dysfunction (moderate to severe vs. none to mild)

312

409

409

409

409

2,012

1,577

110

312

1,010

LV ejection fraction

LV dysfunction

LV dysfunction

NYHA $>$ II

NYHA >ll (vs. no heart failure)

LV dysfunction

LV ejection fraction

$$
<40 \%
$$

LV hypertrophy

LV hypertrophy

Mitral valve prolapse

1,010

Mitral valve regurgitation

Mitral stenosis

312

Mitral annular calcification

290
Mitral valve regurgitation ( $\leq$ mild vs. $\geq$ moderate)
NA NA

NA NA

NA NA

NA NA

NA NA

$14 \% \quad 5 \%$

NA NA

NA NA

NA

NA

NA

NA

NA

NA

NA

NA

NA

NA

NA

NA

NA

NA

$2.4 \%$

NA

NA

NA

$2.3 \%$

NA

NA

NA

NA

NA

NA

NA

NA

NA

NA

NA

NA

NA

NA

NA

NA

NA

$0.8 \%$

NA

NA

NA

NA

NA

NA

NA

NA

$2.1 \%$

NA

NA

NA

NA

NA

NA

NA

NA

OR $0.72(0.27-1.88)$

HR 0.78 (0.36-1.68)

OR 1.611

(1.299-1.999)

RR $1.26(1.11-1.42) \quad<0.05$

RR $2.0(1.0-4.0) \quad 0.05$

$1.8(1.2-3.0) \quad 0.02$

RR $2.5(1.5-4.4) \quad<0.001$

\section{RR 1.795}

(1.214-2.653)

0.003

NA

NS

NA

NA

NA

NS

NA

$0.34(0.04-2.73)$

OR 6.56

RR 2.792

(1.784-4.370)

NA

NS

NS

NA

NA

RR 1.98 (1.09-3.58)

0.025

NA

RR 1.210

(0.774-1.892)

$25 \%$

$9 \%$

OR $0.45(0.2-0.97)$

$<0.05$

(Table 4 continued the next page.) 


\begin{tabular}{|c|c|c|c|c|c|c|}
\hline Study & $\mathbf{n}$ & SHD definition & $\begin{array}{l}\text { Stroke } \\
\text { rate test }\end{array}$ & $\begin{array}{l}\text { Stroke } \\
\text { rate ref. }\end{array}$ & $\begin{array}{l}\text { RR/OR/HR } \\
(95 \% \mathrm{Cl})\end{array}$ & $P$ value \\
\hline Hobbs FD, $2011^{30}$ & 665 & Valvular disease & NA & NA & HR $0.96(0.58-3.13)$ & NS \\
\hline Van Staa TP, $2011^{25}$ & 79,844 & Valvular disease & NA & NA & RR 1.65 (1.01-2.71) & $<0.05$ \\
\hline Aronow WS, $1989^{37}$ & 110 & Left atrial enlargement & NA & NA & OR 4.91 & $<0.05$ \\
\hline SPAF Investigators, $1992^{40}$ & 568 & $\begin{array}{l}\text { Left atrial enlargement } \\
\left.\text { (size } 2.5 \mathrm{vs} .2 .0 \mathrm{~cm} / \mathrm{m}^{2}\right)\end{array}$ & NA & NA & RR $1.6(1.0-2.5)$ & 0.04 \\
\hline \multicolumn{7}{|l|}{ (B) } \\
\hline Study & $\mathbf{n}$ & $\begin{array}{l}\text { (Predisposing) cardio- } \\
\text { vascular condition }\end{array}$ & $\begin{array}{l}\text { Stroke } \\
\text { rate test }\end{array}$ & $\begin{array}{l}\text { Stroke } \\
\text { rate ref. }\end{array}$ & $\begin{array}{l}\text { RR/OR/HR } \\
(95 \% \mathrm{Cl})\end{array}$ & $P$ value \\
\hline Go AS, $2009^{86}$ & 10,908 & Proteinuria & NA & NA & HR 1.54 (1.29-1.85) & $<0.05$ \\
\hline Lin LY, $2011^{21}$ & 7,920 & Chronic kidney disease & NA & NA & $\begin{array}{c}\text { OR } 1.066 \\
(0.834-1.361)\end{array}$ & NS \\
\hline Ruigomez A, $2009^{23}$ & 831 & $\begin{array}{l}\text { Peripheral vascular } \\
\text { disease }\end{array}$ & NA & NA & RR $2.6(1.3-5.1)$ & $<0.05$ \\
\hline Lip, $2010^{15}$ & 1,577 & Vascular disease & $3.6 \%$ & $1.5 \%$ & OR $2.27(0.94-5.46)$ & 0.063 \\
\hline Lin LY, $2011^{21}$ & 7,920 & $\begin{array}{l}\text { Peripheral artery } \\
\text { disease }\end{array}$ & NA & NA & OR 1.81 (1.19-2.77) & 0.006 \\
\hline \multirow[t]{2}{*}{ Rasmussen LH, $2011^{44}$} & 57,053 & Vascular disease & $20.8 \%$ & $8.0 \%$ & $\begin{array}{l}\text { HR } 1.91 \text { (1.44-2.54) } \\
\text { (Stroke or death) }\end{array}$ & $<0.05$ \\
\hline & & Vascular disease & $8.6 \%$ & $4.6 \%$ & $\begin{array}{l}\text { HR } 0.80(0.53-1.20) \\
\text { (Stroke) }\end{array}$ & NS \\
\hline \multirow[t]{2}{*}{ Ruigomez A, $2009^{23}$} & 831 & $\begin{array}{l}\text { Alcohol abuse }(10-19 \\
\text { vs. } 0-1 \text { units/week) }\end{array}$ & NA & NA & RR $0.7(0.2-2.2)$ & NS \\
\hline & & $\begin{array}{l}\text { Alcohol abuse }(\geq 20 \\
\text { units/week vs. } 0-1)\end{array}$ & NA & NA & RR $0.3(0.1-2.4)$ & NS \\
\hline Aronow, $1998^{38}$ & 312 & BMI (obesity) & NA & NA & $\begin{array}{c}\text { RR } 1.093 \\
(0.565-2.114)\end{array}$ & NS \\
\hline \multirow[t]{2}{*}{ Ruigomez A, 2009²3 } & 831 & $\begin{array}{l}\text { BMI (25-29.9 vs. } 20- \\
\left.24.9 \mathrm{~kg} / \mathrm{m}^{2}\right)\end{array}$ & NA & NA & RR $1.0(0.6-2.3)$ & NS \\
\hline & & $\begin{array}{l}\text { BMI }(\geq 30 \text { vs. } 20- \\
\left.24.9 \mathrm{~kg} / \mathrm{m}^{2}\right)\end{array}$ & NA & NA & RR $0.7(0.3-1.9)$ & NS \\
\hline Van Staa TP, $2011^{25}$ & 79,844 & $\begin{array}{l}\mathrm{BMl}<20 \text { vs. } 20- \\
25.9 \mathrm{~kg} / \mathrm{m}^{2}\end{array}$ & NA & NA & RR $1.31(1.06-1.61)$ & $<0.05$ \\
\hline Laupacis A, $1994^{4}$ (AF Investigators) & 1,593 & $\begin{array}{l}\text { Smoking status (smoker } \\
\text { vs. non-smoker) }\end{array}$ & NA & NA & NA & NS \\
\hline \multirow[t]{2}{*}{ Ruigomez A, $2009^{23}$} & 831 & $\begin{array}{l}\text { Smoking status (smoker } \\
\text { vs. non-smoker) }\end{array}$ & NA & NA & RR $1.6(0.7-3.7)$ & NS \\
\hline & & $\begin{array}{l}\text { Smoking status (ex- } \\
\text { smoker vs. non-smoker) }\end{array}$ & NA & NA & RR $0.9(0.4-2.4)$ & NS \\
\hline Ruigomez A, $2009^{23}$ & 831 & Hyperlipidemia & NA & NA & RR $1.2(0.4-3.4)$ & NS \\
\hline Petersen $\mathrm{P}, 1990^{32}$ & 336 & DM & NA & NA & LR Enter 0.16 & NS \\
\hline Laupacis A, $1994^{4}$ (AF Investig. 1994) & 1,593 & DM & NA & NA & RR 1.7 & $<0.05$ \\
\hline $\begin{array}{l}\text { Ezekowitz M, } 1998^{34} \text { (using clinical } \\
\text { factors alone) }\end{array}$ & 1,066 & DM & NA & NA & RR $1.7(1.0-2.8)$ & 0.05 \\
\hline $\begin{array}{l}\text { Ezekowitz M, } 1998^{34} \text { (using clinical and } \\
\text { echocardiographic features) }\end{array}$ & 1,010 & DM & NA & NA & RR $1.7(1.0-2.9)$ & 0.05 \\
\hline Aronow WS, $1998^{38}$ & 312 & DM & NA & NA & RR $0.83(0.56-1.22)$ & NS \\
\hline Seidl K, $1998^{42}$ & 191 & DM & NA & NA & NA & NS \\
\hline SPAF III, $1998^{27}$ & 892 & DM & NA & NA & NA & NS \\
\hline Stollberger CM, $1998^{35}$ & 490 & DM & NA & NA & NA & NS \\
\hline Hart RG, $1999^{28}$ & 2,012 & DM & NA & NA & $\begin{array}{l}\text { RR } 1.9 \text { (for disabling/ } \\
\text { fatal stroke) }\end{array}$ & 0.02 \\
\hline \multirow[t]{2}{*}{ Wang TJ, $2003^{36}$} & 705 & DM & NA & NA & $\begin{array}{l}\text { HR } 1.80 \text { (1.43-3.13) } \\
\text { (for stroke) }\end{array}$ & $<0.05$ \\
\hline & & & NA & NA & $\begin{array}{l}\text { HR } 1.40 \text { (1.07-1.83) } \\
\text { (for stroke or death) }\end{array}$ & $<0.05$ \\
\hline Lip GYH, $2010^{15}$ & 1,577 & DM & $4.3 \%$ & $1.9 \%$ & OR $1.79(0.73-4.40)$ & NS \\
\hline Hobbs FD, $2011^{30}$ & 665 & DM & NA & NA & HR 2.06 (1.07-3.98) & 0.03 \\
\hline Lin LY, $2011^{21}$ & 7,920 & DM & NA & NA & $\begin{array}{c}\text { OR } 1.341 \\
(1.092-1.648)\end{array}$ & 0.005 \\
\hline Van Staa TP, $2011^{25}$ & 79,844 & DM & NA & NA & RR 1.33 (1.14-1.55) & $<0.05$ \\
\hline
\end{tabular}

BMI, body mass index; CHF, congestive heart failure; DM, diabetes mellitus; NYHA, New York Heart Association heart failure stage; LV, left ventricular; MI, myocardial infarction; SHD, structural heart disease. Other abbreviations as in Tables 1,2. 
Table 6. Published Risk Stratification Models

(A) Reviewed models

RSM (year)

ACCP (1998)

ACCP (2004)

ACCP (2008)

AFI (1994)

AFI (1996)

AFI (2003)

AHA/ACC/ESC (2001)

AHA/ACC/ESC (2006)

$\mathrm{CHADS}_{2}$ (2001)

EAFT (1995)

Framingham (2003)

SPAF (1992)

SPAF (1995)

SPAF (1998)

SPAF (1999)

SPAF (1999)

Revised CHADS2 (2008)

NICE guidelines (2006)

$\mathrm{CHA}_{2} \mathrm{DS}_{2}$-VASc (2010)
Ref.

Laupacis A, 1998

Singer DE, 200487

Singer DE, 2008

Laupacis A, 19944

Lip GYH, $1996^{88}$

vanWalraven $C, 2003^{51}$

Fuster V, 200147

Fuster V, $2006^{89}$

Gage BF, $2001^{49}$

Van Latum JC, $1995^{24}$

Wang TJ, $2003^{36}$

SPAF Investigators,

$1992^{40}$

SPAF Investigators, $1995^{41}$

Ezekowitz, $1998^{27}$

Hart RG, $2000^{29}$

Hart RG, $1999^{28}$

Rietbrock S, $2008^{90}$

Lip GYH, $2006^{91}$

Lip GYH, 201015

\begin{tabular}{c} 
Derivation \\
Consensus \\
Consensus \\
Consensus \\
Consensus \\
Consensus \\
Evidence \\
Consensus \\
Consensus \\
Consensus \\
Evidence \\
Evidence \\
Evidence \\
Evidence \\
\hline Evidence \\
Evidence \\
Evidence \\
Consensus \\
Evidence \\
Evidence
\end{tabular}

Derivation

Stratification

3 categories

3 categories

3 categories

2 categories $^{\star \star}$

3 categories

2 categories

2 categories

3 categories

Score 0-6

3 categories

Score 0-31

3 categories

2 categories

3 categories

3 categories

3 categories

Score (max. 14)

Algorithm based

approach

Score 0-9
Derivation population/ organization

ACCP (1998) ${ }^{\dagger}$

ACCP (2004)

ACCP (2008)

AFASAK, BATAAF,

CAFA, SPAF, SPINAF

None $^{*}$

AHA/ACC/ESC (2001)

AHA/ACC/ESC (2001)

AHA/ACC/ESC (2006)

None*

EAFT

Framingham Heart Study SPAF I

SPAF II, SPAF II

SPAF III

SPAF III

SPAF I, SPAF II, SPAF III

GPRD

SPAF III

SPAF III, Euro Heart Survey

\section{(B) Stroke risk factors included in each RSM}

\begin{tabular}{|c|c|c|c|c|c|c|c|c|c|}
\hline RSM (year) & AF duration & Age & DM & Female & Htn & Vasc & SHD & Stroke/TE & Thyrotox \\
\hline ACCP (1998) & - & + & + & - & + & - & + & - & + \\
\hline ACCP $(2004)^{87}$ & - & + & + & - & + & - & + & + & - \\
\hline AFI $(1994)^{4}$ & - & - & + & - & + & - & - & + & - \\
\hline AFI (1999) & - & + & + & - & + & - & - & + & - \\
\hline AFI $(2003)^{51}$ & - & - & + & - & + & - & + & + & - \\
\hline $\begin{array}{l}\text { AHA/ACC/ESC } \\
(2001)^{47}\end{array}$ & - & + & + & - & + & - & + & - & + \\
\hline CHADS2 $(2001)^{49}$ & - & + & + & - & + & - & + & + & - \\
\hline EAFT $(1995)^{24}$ & + & + & - & - & + & - & + & + & - \\
\hline Framingham (2003) ${ }^{36}$ & - & + & + & + & + & - & - & - & - \\
\hline SPAF $(1992)^{40}$ & - & - & - & - & + & - & + & + & - \\
\hline SPAF $(1995)^{41}$ & - & + & - & + & + & - & + & + & - \\
\hline SPAF $(1998)^{27}$ & - & + & - & + & + & - & + & + & - \\
\hline SPAF $(1999)^{29}$ & - & + & + & + & + & - & - & - & - \\
\hline SPAF $(1999)^{28}$ & - & + & + & + & + & - & - & - & - \\
\hline $\begin{array}{l}\text { Rietbrock-CHADS } 2 \\
(2008)^{90}\end{array}$ & - & + & + & + & + & - & - & + & - \\
\hline $\begin{array}{l}\mathrm{CHA}_{2} \mathrm{DS}_{2}-\mathrm{VASc} \\
(2010)^{15}\end{array}$ & - & + & + & + & + & + & + & + & - \\
\hline
\end{tabular}

(C) Summary of results of model evaluation studies in terms of reported C statistics regarding prediction of stroke

\begin{tabular}{|c|c|c|c|c|c|}
\hline RSM & Gage BF, 200149 & Gage BF, 2004 ${ }^{52}$ & Lip, GYH, 2010'15 & Olesen JB, $2011^{16}$ & Hobbs FD, $2011^{30}$ \\
\hline ACCP (1998) & NR & 0.58 & NR & NR & NR \\
\hline ACCP (2008) & NA & NA & 0.57 & NR & 0.60 \\
\hline AFI $(1994)^{4}$ & 0.68 & 0.63 & 0.57 & NR & NR \\
\hline $\mathrm{CHADS}_{2}(2001)^{49}$ & 0.82 & 0.70 & 0.56 & 0.72 & 0.55 \\
\hline Framingham $(2003)^{36}$ & NR & 0.69 & 0.64 & NR & 0.59 \\
\hline SPAF $(1998)^{27}$ & NR & 0.64 & $N R$ & NR & NR \\
\hline SPAF $(1999)^{29}$ & 0.74 & NR & 0.55 & NR & NR \\
\hline $\mathrm{AHA} / \mathrm{ACC} / \mathrm{ESC}(2006)^{89}$ & NA & NA & 0.57 & NR & 0.61 \\
\hline Rietbrock-CHADS $2(2008)^{90}$ & NA & NA & 0.59 & NR & 0.62 \\
\hline $\mathrm{CHA}_{2} \mathrm{DS}_{2}-\mathrm{VASc}(2010)^{15}$ & NA & NA & 0.61 & 0.85 & 0.60 \\
\hline
\end{tabular}


${ }^{*}$ Risk factors were identified from other stratification schemes. ${ }^{* *}$ Each category applied to 3 different age ranges, although in the AFI 2 and AFI 3 models, age was incorporated as a risk factor. ${ }^{\dagger}$ Although the recommendations of each ACCP guideline supersede the previous version, the 1998 version contained an RSM that was externally validated, and is referenced only for this reason.

GPRD, General Practice Research Database; SHD, structural heart disease (including MI and angina); RSM, risk stratification model; TE, thromboembolism; Thyrotox, thyrotoxicosis. Other abbreviations as in Tables 1-4. [number] reference number.

bolism.

The systematic search of papers evaluating both internally and externally validated stroke risk models resulted in 12 studies, ${ }^{4,28,40,41,43,49-52}$ following exclusion of 1 study because of substratification by age group. ${ }^{4}$ In total, 12 models were evaluated on 28 occasions.

When considering the $95 \%$ confidence intervals (CIs) for the observed stroke incidence of each risk category for each RSM, only one stroke risk model using 3 risk strata (low, moderate, high) reached separation of each risk group at the $95 \%$ CI level; that is, the Stroke Prevention in AF model. ${ }^{28}$ The results of the studies found to have reported on discriminative capabilities of each RSM using the c-statistic are shown in Table 6C.

One recent study reported the values of the c-statistic for 9 RSMs within the same population and found the Rietbrock modified $\mathrm{CHADS}_{2}$-index to have the highest c-statistic. ${ }^{30}$ The $\mathrm{CHADS}_{2}$ score was found to have the highest c-statistic in the same population in another study comparing 5 different RSMs. ${ }^{52}$ The largest of all comparative studies, a nationwide cohort, found that the highest c-statistic for an evaluation of 2 RSMs using the same population was for the $\mathrm{CHA}_{2} \mathrm{DS}_{2}-\mathrm{VASc}$ score. ${ }^{16}$ The various risk stratification schemes classified variable proportions as low, moderate and high risk, but in general, the $\mathrm{CHA}_{2} \mathrm{DS}_{2}$-VASc score classified the lowest proportion of patients as 'low risk'.

\section{Cost-Effectiveness}

An overview of studies looking into the cost-effectiveness of antithrombotic therapy for AF can be found in Table 7.

Vitamin K Antagonists vs. No Anticoagulation Anticoagulation treatment in a general AF population was found to be relatively cost-effective compared to no anticoagulation in all 9 studies reporting on it. ${ }^{53-61}$ The incremental cost-effectiveness rate (ICER) in a British study ranged from $£ 1,751$ and $£ 13,221$ per life-year gained free from stroke, ${ }^{56}$ which is comparable to reports from North America and Sweden during the same time period, reporting, US\$1,907 and SKR171,000417,000 , respectively. The latter range depended on the perceived risk of bleeding being either low $(0.3 \%)$ or more average $(2 \%)$.

Caro et al found an annual reduction in total healthcare costs of US\$1,514 if anticoagulation was used in AF patients. ${ }^{53}$ Cost-effectiveness of VKAs also depends on the risk of stroke. In patients at low risk (ie, only $\mathrm{AF}$ as a stroke risk factor), there is no perceived benefit when they are treated with VKAs, expressed by the increased costs and decreased quality-adjusted life years (QALY), whereas patients with more than 2 of the classical risk factors of stroke do benefit, with an ICER of US $\$ 1,434$ - £6,000 - per QALY..$^{54,55,57,60}$

Antiplatelet Therapy vs. Vitamin K Antagonists A UK study in a high risk, elderly ( $\geq 75$ years) population found the total costs, associated with both hemorrhagic and thrombotic events, to be lower and QALY scores to be higher in patients receiving warfarin compared to those receiving aspirin. ${ }^{62} \mathrm{An}$ other study in less elderly AF patients with a 'moderate' to high stroke risk also demonstrated the cost-effectiveness of vitamin $\mathrm{K}$ antagonists over aspirin (ICER US\$500/QALY). ${ }^{55}$
Three other studies supported the cost-effectiveness of VKAs compared with aspirin. ${ }^{53,54,61}$ In addition to the stroke risk reduction playing an important role in treatment decisions, the quality of anticoagulation and discontinuation of the drug should also be considered. ${ }^{59}$ The benefits for patients at low risk of stroke comes at the price of significantly increased costs over aspirin (US\$370,000/QALY in 65-year-old patients and US\$110,000/QALY in 75-year-old patients) compared to those at medium to high stroke risk (US\$8,000/QALY). Also, bleeding risk was found to have a negative effect on the cost-effectiveness of VKAs. ${ }^{55}$

Dabigatran vs. Vitamin K Antagonists or Antiplatelet Therapy Three studies reported on the cost-effectiveness of the new oral direct thrombin inhibitor, dabigatran, compared to other forms of antithrombotic therapy. ${ }^{63-65}$ One study found that, assuming an average risk of major bleeding, only aspirin was cost-effective for those at lowest risk of stroke $\left(\mathrm{CHADS}_{2}\right.$ score $=0)$. Unless the TTR was poor $(<57.1 \%)$ or the risk of major bleeding increased, warfarin was cost-effective in those at moderate risk of stroke ( $\mathrm{CHADS}_{2}$ score 1 or 2). Dabigatran $150 \mathrm{mg}$ twice daily was cost-effective in those with a $\mathrm{CHADS}_{2}$ score $>2$, when the TTR was not excellent $(>72.6 \%) .{ }^{64}$ A lower dose (110 mg twice daily) of dabigatran and clopidogrel, in addition to aspirin, was not found to be cost-effective in this analysis. ${ }^{64}$ From a Canadian perspective, dabigatran etexilate was found to be cost-effective compared to VKAs (ICER $\$ 10,440 / \mathrm{QALY}$ ) in patients at moderate to high risk of stroke. ${ }^{65}$ Another study found the price of dabigatran to be of importance, as the assumption of US\$13,80 for the high (150 twice daily) dose resulted in an ICER of US\$51,229/QALY for lowdose dabigatran and US\$ 45,372/QALY for high-dose dabigatran. ${ }^{63}$

\section{Discussion}

This systematic review demonstrates that the strongest, independent predictors of stroke are a prior stroke or TIA, age $>75$ years, hypertension, diabetes mellitus and structural heart disease. Sex, heart failure and vascular disease cause more discussion because the available evidence favoring thier use as risk factors in AF per se is not as convincing. The interplay of less powerful independent risk factors of stroke could potentially help explain apparent differences in predictive ability of (clinical) entities such as, for instance, heart failure.

Whereas the more clinical diagnosis of CHF fails to be an independent risk factor for stroke in AF patients, a more objective/quantitative approach (eg, LVD) does prove more consistently to be an independent stroke risk factor, but not always. ${ }^{66}$ Although almost being ignored in the historical anticoagulation trials of 2 decades ago, most studies identified by our search and the nearly all of the most recent available epidemiological evidence clearly indicate the importance of vascular disease as an significant stroke risk predictor. ${ }^{66-71}$ However, it is important to further differentiate vascular disease (eg, aortic disease, peripheral vs. coronary artery disease or myocardial infarction), as the former seems to be the driving force behind vascular disease being an independent stroke risk factor in AF patients. ${ }^{70}$ 


\begin{tabular}{|c|c|c|c|c|}
\hline Study & Comparison & Population & QALY & ICER \\
\hline \multirow[t]{4}{*}{$\begin{array}{l}\text { Gage BG, } \\
1995^{55}\end{array}$} & \multirow[t]{4}{*}{$\begin{array}{l}\text { VKA vs. no } \\
\text { OAC }\end{array}$} & \multirow[t]{4}{*}{$\begin{array}{l}\text { Nonvalvular AF (NVAF). Base case: } \\
\text { male, age } 65 \text { years, good candidates } \\
\text { for warfarin. Risk factors: prior stroke } \\
\text { or TIA, diabetes, Htn, heart disease }\end{array}$} & $\begin{array}{l}\text { QALYs during next } 10 \text { years for } \\
\text { base case }\end{array}$ & $\begin{array}{l}\text { Costs in } 1994 \text { US } \$ \text { of prophylaxis, stroke, } \\
\text { hemorrhage, and death in next } 10 \text { years. } \\
\text { Marginal cost-effectiveness per QALY } \\
\text { saved with warfarin during next } 10 \text { years } \\
\text { (MCE) }\end{array}$ \\
\hline & & & $\begin{array}{l}\text { [High risk] Warfarin: } 6.51 \text {, No } \\
\text { therapy: } 6.01\end{array}$ & $\begin{array}{l}\text { Cost } \$ 12,500 \text {, Cost } \$ 15,300 \text {, MCE: Warfa- } \\
\text { rin preferred to no therapy }\end{array}$ \\
\hline & & & $\begin{array}{l}\text { [Medium risk] Warfarin: } 6.70 \text {, No } \\
\text { therapy: } 6.23\end{array}$ & $\begin{array}{l}\text { Cost } \$ 10,900 \text {, Cost } \$ 11,400, \text { MCE: Warfa- } \\
\text { rin preferred to no therapy }\end{array}$ \\
\hline & & & $\begin{array}{l}\text { [Low risk] Warfarin: } 6.70 \text {, No } \\
\text { therapy: } 6.51\end{array}$ & $\begin{array}{l}\text { Cost } \$ 9,000 \text {, Cost } \$ 6,300 \text {, MCE: } \$ 14,000 \\
\text { (range } 7,700-24,000 \text { ) vs. no therapy }\end{array}$ \\
\hline \multirow[t]{2}{*}{$\begin{array}{l}\text { Caro JJ, } \\
1997^{53}\end{array}$} & \multirow[t]{2}{*}{$\begin{array}{l}\text { VKA vs. no } \\
\text { OAC }\end{array}$} & \multirow[t]{2}{*}{ NVAF } & & $\begin{array}{l}\text { Costs per year in } 1995 \text { US\$ of prophy- } \\
\text { laxis, emboli and bleeding }\end{array}$ \\
\hline & & & $\begin{array}{l}\text { Warfarin: NR, No prophylaxis: } \\
\text { NR }\end{array}$ & $\begin{array}{l}\text { Cost } \$ 2,599 \text {, Cost } \$ 4,113 \text {, Net saving } \\
\$ 1,514\end{array}$ \\
\hline \multirow[t]{2}{*}{$\begin{array}{l}\text { Eckman MH, } \\
1998^{54}\end{array}$} & \multirow[t]{2}{*}{$\begin{array}{l}\text { VKA vs. no } \\
\text { OAC }\end{array}$} & \multirow[t]{2}{*}{$\begin{array}{l}\text { NVAF. Base case: } 69 \text {-year-old man, } \\
\text { prototypical of } 5 \text { clinical trials consid- } \\
\text { ered }\end{array}$} & QALY & $\begin{array}{l}\text { Costs over lifetime in } 1998 \text { US\$, including } \\
\text { systemic embolism, bleeding and associ- } \\
\text { ated morbidity. Marginal cost-effective- } \\
\text { ness ratio (MCER, \$ per additional QALY) }\end{array}$ \\
\hline & & & $\begin{array}{l}\text { Warfarin: } 7.60, \text { No prophylaxis: } \\
7.02\end{array}$ & $\begin{array}{l}\text { Cost } \$ 13,812 \text {, Cost } \$ 12,704, \text { MCER } \\
\$ 1,907\end{array}$ \\
\hline \multirow[t]{5}{*}{$\begin{array}{l}\text { Lightowlers S, } \\
1998^{56}\end{array}$} & \multirow[t]{5}{*}{$\begin{array}{l}\text { VKA vs. no } \\
\text { OAC }\end{array}$} & \multirow{5}{*}{$\begin{array}{l}\text { NVAF. Base cases } 1 \text { and } 2 \text { from } \\
\text { BAATAF trial } 4 \text {-year data: case } 1 \text {, } \\
\text { hazard rates remained constant from } \\
\text { end of } 4 \text { th year to } 10 \text { th year; case } 2 \text {, } \\
\text { mean hazard rates for years } 1-4 \\
\text { remained for years } 5-10 \text {. Cases } 3 \text { and } \\
4 \text { from meta-analysis data: case } 3 \text { used } \\
\text { hazard rates for the whole group; case } \\
4 \text { used hazard rates for the group aged } \\
>75 \text { years }\end{array}$} & $\begin{array}{l}\text { Life-years gained (discounted) } \\
\text { free from stroke over 10-year } \\
\text { period with warfarin vs. no } \\
\text { prophylaxis }\end{array}$ & $\begin{array}{l}\text { Incremental cost-effectiveness ratio } \\
\text { (ICER, cost per life-year gained, [benefits } \\
\text { discounted]) in } 1997 \text { UK£, including anti- } \\
\text { coagulation, stroke and bleeding }\end{array}$ \\
\hline & & & [Case 1] 0.33061 & ICER £13,221.29 \\
\hline & & & [Case 2] 0.5349 & ICER £5,497.59 \\
\hline & & & [Case 3] 0.9349 & ICER $£ 1,751.05$ \\
\hline & & & [Case 4] 1.908 & ICER -£400.45 \\
\hline \multirow[t]{2}{*}{$\begin{array}{l}\text { Thomson R, } \\
2000^{60}\end{array}$} & \multirow[t]{2}{*}{$\begin{array}{l}\text { VKA vs. no } \\
\text { OAC }\end{array}$} & \multirow{2}{*}{$\begin{array}{l}\text { NVAF. } 12 \text { groups were defined accord- } \\
\text { ing to combinations of sex and age. } \\
\text { Overall, } 1,512 \text { combinations of sex, } \\
\text { age, systolic blood pressure, and other } \\
\text { risk factors were assessed }\end{array}$} & QALY & $\begin{array}{l}\text { Cost per QALY gain in UK£, including } \\
\text { stroke, warfarin treatment, Gl bleeding }\end{array}$ \\
\hline & & & NA & $\begin{array}{l}\text { In most cases, treatment led to lower } \\
\text { costs. In } 12 / 1,512 \text { combinations }(0.8 \%) \\
\text { there was a cost per QALY gained in the } \\
\text { range } £ 250-6,000\end{array}$ \\
\hline \multirow[t]{5}{*}{$\begin{array}{l}\text { Desbiens NA, } \\
2002^{57}\end{array}$} & \multirow[t]{5}{*}{$\begin{array}{l}\text { VKA vs. no } \\
\text { OAC }\end{array}$} & \multirow[t]{5}{*}{$\begin{array}{l}\text { NVAF. Assessed according to age and } \\
\text { additional risk factors }\end{array}$} & QALY & $\begin{array}{l}\text { Costs in US\$ and MCER (\$ per additional } \\
\text { QALY) }\end{array}$ \\
\hline & & & $\begin{array}{l}\text { [Low risk (age } 65 \text { years+no risk } \\
\text { factors)] Anticoagulation: } 11.5, \\
\text { No anticoagulation: } 11.8\end{array}$ & NA \\
\hline & & & $\begin{array}{l}\text { [High risk (age } 65 \text { years, prior } \\
\text { stroke or TIA, DM and Htn)] Anti- } \\
\text { coagulation: } 11.7 \text {, No anticoagu- } \\
\text { lation: } 9.5\end{array}$ & Cost $-\$ 1,434$ and 2.2 QALY gained \\
\hline & & & $\begin{array}{l}\text { [High risk (age } 85 \text { years, prior } \\
\text { stroke or TIA, DM and Htn)] }\end{array}$ & Cost $-\$ 1,767$ and 0.5 QALY gained \\
\hline & & & $\begin{array}{l}\text { [High risk (age } 95 \text { years, prior } \\
\text { stroke or TIA and Htn)] }\end{array}$ & MCER $\$ 30,000$ per QALY gained \\
\hline $\begin{array}{l}\text { Sorensen SV, } \\
2009^{59}\end{array}$ & & $\begin{array}{l}\text { NVAF at moderate to high risk of } \\
\text { stroke. Base case age } 70 \text { years. } \\
\text { Scenario 1: all patients start on warfa- } \\
\text { rin and INR values are always in range. } \\
\text { Scenario 2: all patients start on warfa- } \\
\text { rin with trial-like INR control. Scenario } \\
\text { 3: all patients start on warfarin with } \\
\text { real-world INR control. Scenario 4: } \\
\text { real-world prescription (and control) of } \\
\text { warfarin, aspirin, or neither for warfa- } \\
\text { rin-eligible patients }\end{array}$ & QALY & $\begin{array}{l}\text { Costs over lifetime in } 2006 \text { US\$, including } \\
\text { drug and monitoring, acute events, long- } \\
\text { term care }\end{array}$ \\
\hline $\begin{array}{l}\text { Sorensen SV, } \\
2009^{59}\end{array}$ & $\begin{array}{l}\text { VKA perfect } \\
\text { INR vs. trial } \\
\text { INR }\end{array}$ & & $\begin{array}{l}\text { Scenario } 2 \text { (trial-like INR): } 6.92 \text {, } \\
\text { Scenario } 1 \text { (perfect INR): } 7.21\end{array}$ & $\begin{array}{l}\text { Cost } \$ 77,764 \text {, Cost } \$ 68,039, \text { Scenario } 1 \\
\text { vs. 2: Cost } \$ 5,981-13,775 \text { and } 0.38 \\
-0.76 \text { QALY gained }\end{array}$ \\
\hline $\begin{array}{l}\text { Sorensen SV, } \\
2009^{59}\end{array}$ & $\begin{array}{l}\text { VKA real- } \\
\text { world INR vs. } \\
\text { trial INR }\end{array}$ & & $\begin{array}{l}\text { Scenario } 2 \text { (trial-like INR): } 6.92 \text {, } \\
\text { Scenario } 3 \text { (real-world INR): } \\
6.75\end{array}$ & $\begin{array}{l}\text { Cost } \$ 77,764, \text { Cost } \$ 84,518, \text { Scenario } 3 \\
\text { vs. 2: Cost } \$ 3,699-9,970 \text { and }-0.23-0.57 \\
\text { QALY gained }\end{array}$ \\
\hline $\begin{array}{l}\text { Sorensen SV, } \\
2009^{59}\end{array}$ & $\begin{array}{l}\text { Real-world } \\
\text { prescription } \\
\text { (and INR) vs. } \\
\text { trial INR }\end{array}$ & & $\begin{array}{l}\text { Scenario } 2 \text { (trial-like INR): } 6.92 \text {, } \\
\text { Scenario } 4 \text { (real-world warfarin, } \\
\text { aspirin, or no treatment): } 6.67\end{array}$ & $\begin{array}{l}\text { Cost } \$ 77,764 \text {, Cost } \$ 87,248 \text {, Scenario } 4 \\
\text { vs. } 2 \text { : Cost } \$ 4,400-13,901 \text { and }-0.41 \\
-0.86 \text { QALY gained }\end{array}$ \\
\hline
\end{tabular}




\section{Study \\ Comparison}

Mercaldi CJ, VKA vs. no $2011^{61}$
Population

NVAF patients in the Centers for Medicare and Medicaid Services 5\% Sample Standard Analytic Files

NVAF Base case: age 65 years, good candidate for warfarin. Risk factors: Prior stroke or TIA, diabetes, Htn, heart disease
VKA vs. AP Gage

Eckman MH, VKA vs. AP $1998^{54}$

NVAF. Base case: 69 -year-old man prototypical of 5 clinical trials considered

Jowett $S, \quad$ VKA vs. AP $A F, \geq 75$ years of age $2011^{62}$

Caro JJ, $\quad$ AP vs. no NVAF $1997^{53}$ OAC

$\begin{array}{ll}\text { Freeman JV, } & \text { Dabigatran } \\ 2011^{63} & \text { vs. VKA }\end{array}$

Sorensen SV, Dabigatran $2011^{65}$ vs. VKA

Shah SV, Dabigatran vs. AP

Patients NVAF aged $\geq 65$ years and with risk factors for stroke (CHADS $_{2}$ score $\geq 1$ or equivalent) and no contraindications to anticoagulation

Shah SV, Dabigatran Hypothetical cohort of 70-year-old $2011^{64}$ Vs. VKA

$2011^{64}$ patients with AF who had a moderate risk of stroke and no contraindication to anticoagulant therapy, based on the RE-LY trial

$\mathrm{AF}$ and $\geq 1$ additional risk factor for stroke, or AF and impaired LVEF; eligible for anticoagulation. Evaluated DE in a sequential dosing approach, with patients $<80$ years of age receiving $150 \mathrm{mg}$ bid and patients $\geq 80$ receiving $110 \mathrm{mg}$ bid. Comparisons made with warfarin with trial-like INR control or real-world prescription (and control) of warfarin, aspirin, or neither

Hypothetical cohort of 70-year-old patients with AF who had a moderate risk of stroke and no contraindication to anticoagulant therapy, based on the RE-LY trial
Warfarin: 7.60, Aspirin: 7.20

QALY

Warfarin: NA, No warfarin: NA

QALYs during next 10 years for 65-year-old with NVAF

[High risk ( $+\geq 2$ risk factors)] Warfarin: 6.51, Aspirin: 6.27 [Medium risk ( $+\geq 1$ risk factor)] Warfarin: 6.60, Aspirin: 6.46 [Low risk (age 60-69 years + no risk factors)]

Warfarin: 6.70, Aspirin: 6.69

QALY

QALY over 4 years

Aspirin: 1.665, Warfarin: 1.685, Difference 0.020 (95\% Cl -0.070 to 0.111 ) favoring warfarin (NS)

Aspirin: NR, Aspirin: NR, No prophylaxis: NR

QALY

\section{ICER}

Costs in 2006 US\$ per patient per year, including events, inpatient and outpatient utilization

Cost $\$ 18,621$ (SD 38,249), Cost $\$ 22,135$ (SD 46,048), Difference $-\$ 9,836$ $(\mathrm{P}<0.0001)$

Marginal cost-effectiveness per QALY saved with warfarin during next 10 years (MCE). Costs in 1994 US\$ of prophylaxis, stroke, hemorrhage, and death in next 10 years

Cost $\$ 12,500$, Cost $\$ 13,200$, MCE warfarin preferred to aspirin

Cost $\$ 10,900$, Cost $\$ 9,700$, MCE $\$ 8,000$ (range 200-30,000) vs. aspirin

Cost $\$ 9,000$, Cost $\$ 5,400$, MCE $\$ 370,000$ (range 66,000-aspirin preferred) vs. aspirin

Costs over lifetime in 1998 US\$, including systemic embolism, bleeding and associated morbidity. Marginal cost-effectiveness ratio (MCER, \$ per additional QALY) Cost $\$ 13,812$, Cost $\$ 12,020$, MCER $\$ 4,484$

Cost in 1997 UK£ over 4 years, including clinical events, primary care, coagulation clinic visits

Cost £1,548 (SD 2468), Cost £1382 (SD 2004), Difference $-£ 166(95 \% \mathrm{Cl}-452$ to 89) NS

Costs per year in 1995 US\$ of prophylaxis, emboli and bleeding

Cost \$4,313 (excluding SPAF II trial), Cost $\$ 3,139$ (including SPAF II trial), Cost $\$ 4,113$, Net expense $\$ 200$ (excluding SPAF II trial), Net saving $\$ 974$ (including SPAF II trial)

Costs and ICER in 2008 US\$

ICER

Warfarin: 10.28 , DE $110 \mathrm{mg}$ bid: 10.70

Warfarin: 10.28 , DE $150 \mathrm{mg}$ bid:

10.84

QALY

Cost $\$ 143,193$, Cost $\$ 168,398$, ICER $\$ 45,372$

Cost over 20 years and marginal cost per QALY (MCER) in 2010 US\$, including antithrombotic therapy, hemorrhage, neurological ischemia, dyspepsia and $\mathrm{MI}$ Cost $\$ 23,000$, Cost $\$ 43,700$, MCER $\$ 86,000$

Warfarin: 8.40 , DE $150 \mathrm{mg}$ bid: 8.65

Warfarin: 8.40 , DE $110 \mathrm{mg}$ bid: 8.54

Cost $\$ 23,000$, Cost $\$ 44,300$, MCER $\$ 150,000$

Warfarin: 8.40 , Aspirin and clopi- Cost $\$ 23,000$, Cost $\$ 34,000$, MCER dogrel: 8.32

QALY

Base case: Trial-like warfarin:

7.08, Sequential dosing DE:

7.29

Scenario 1: Real-world prescrib-

ing warfarin: 7.01 , Sequential dosing DE: 7.29

Scenario 2: Trial-like warfarin:

6.68, DE $150 \mathrm{mg}$ bid: 6.86

Scenario 3: Trial-like warfarin:

6.68, DE $110 \mathrm{mg}$ bid: 6.82

QALY

Aspirin: 8.17 , DE $150 \mathrm{mg}$ bid 8.65

Aspirin: 8.17 , DE $110 \mathrm{mg}$ bid:

8.54

Aspirin: 8.17 , Warfarin: 8.40

Aspirin: 8.17, Aspirin and clopidogrel: 8.32
Dominated

ICER (2010 Canadian \$ per QALY)

ICER $\$ 10,440$

\section{ICER $\$ 3,962$}

Scenario 2: 9,041

Scenario 3: 29,994 QALY (MCER) in 2010 US\$, including antithrombotic therapy, hemorrhage, neurological ischemia, dyspepsia and MI Cost $\$ 20,000$, Cost $\$ 43,700$, MCER $\$ 50,000$

Cost $\$ 20,000$, Cost $\$ 44,300$, MCER

$\$ 66,000$

Cost $\$ 20,000$, Cost $\$ 23,000$, MCER $\$ 12,500$ $\$ 99,000$
Cost over 20 years and marginal cost per

Cost $\$ 20,000$, Cost $\$ 34,000$, MCER

$\mathrm{DE}$, dabigatran etexilate; NVAF, nonvalvular AF. Other abbreviationas as in Tables 1-5. [number] reference number. 
In recent years, female sex has also been identified as an independent stroke risk factor in some, ${ }^{15,39,72}$ but not all ${ }^{21,25,30}$ studies. Especially in light of recent developments in both stroke risk stratification and the availability of new oral anticoagulants, resulting into adjusted recommendations for the use of oral anticoagulation further studies for this matter are warranted. ${ }^{72}$

Fortunately, readily available clinical risk factors are usually sufficient to adequately commence and review antithrombotic therapy. However, when in doubt, echocardiography can assist. ${ }^{26,34,37,38,41,73}$ Transthoracic echocardiography might show left ventricular hypertrophy, in the absence of aortic valve stenosis possibly reflecting end-organ damage caused be (undetected or masked) hypertension. Similarly, it might show a reduced left ventricular ejection fraction without the (clear) clinical syndrome of heart failure. A transesophageal echocardiogram $^{26,37}$ adds further valuable data because it provides even more (reliable) local information on aspects such as vascular disease (aortic plaques) and structural heart disease (atrial dilatation). However, up to now there has been insufficient recent evidence to support the use of left atrial dimension as a stroke risk factor.

Although the searches identified a few recent studies that considered laboratory data/biomarkers as stroke risk factors, none of those studied parameters was found to be an independent predictor of stroke. Still, there is reason to be optimistic about the future possibilities of biomarkers when considering the prevention of AF-related stroke in the broadest sense. ${ }^{74}$

Regarding the health economics analyses, the quality of the studies were good and suggests that OAC is cost-effective in patients at moderate to high risk of stroke, ${ }^{53-61}$ but that appears not to be so for those low-risk patients, for whom aspirin seems cost-effective, ${ }^{55}$ although the option of no antithrombotic therapy as an alternative in this subset has not been tested. The new oral direct thrombin inhibitor, dabigatran, appears to be cost-effective in high risk patients and also those at moderate risk of stroke; ${ }^{63-65}$ however, actual cost price and the individual TTR may also be important factors that influence the latter consideration. Furthermore, limited data on the other new oral anticoagulants, rivaroxaban and apixaban, have been published recently. ${ }^{75,76}$ Even more data ${ }^{77-79}$ and comparative efforts are awaited to facilitate optimal decision making in the antithrombotic management of AF patients.

We emphasize that the focus of this review was on stroke risk assessment. However, at the same time we also acknowledge the well-known fact that the risk of stroke and major bleeding go, to some extent, hand in hand and the latter therefore also needs to be considered when dealing with antithrombotic therapy.

Recently, a novel user-friendly risk score called HAS-BLED was introduced..$^{80}$ The backbone of this bleeding risk stratification tool was derived from the literature on risk factors of anticoagulation-related to major bleeding which was systematically searched and appraised. ${ }^{81}$ It underwent additional fine-tuning and validation in a large European cohort of $\mathrm{AF}$ patients and further validations have recently been published, ${ }^{82,83}$ contributing to its incorporation into the latest European guidelines on the management of AF. ${ }^{17}$ Another bleeding risk assessment tool, the ATRIA bleeding score ${ }^{84}$ consists of 5 weighted factors, but appears to be less user-friendly and was recently outperformed by the HAS-BLED score regarding predictive ability for major bleeding. ${ }^{83}$ More importantly, the goal of HAS-BLED or any other practical bleeding risk tool is not to provide a cut-off for when not to commence or continue antithrombotic therapy. It serves as a reminder to all physi- cians dealing with antithrombotic drugs in AF patients of the risk factors for major bleeding and hopefully triggers appropriate prevention or active treatment to reduce risk. This approach seems very helpful, given the new oral anticoagulants ${ }^{6-8}$ and the recent evidence, both from clinical' ${ }^{9}$ and 'real-world' data, ${ }^{11,85}$ that further support the limited role of aspirin as a suitable, safe alternative to OAC.

\section{Study Limitations}

The presented review data consists of epidemiological, cohort and clinical studies, all of which were not free from selection bias, to a substantial degree in some cases. Especially regarding the clinical studies, we must keep in mind that only a small minority of the screened patients were actually randomized and therefore included in the data presented. Furthermore, certain conditions (eg, vascular disease) were not consistently recorded or taken into account. Altogether, this could very well affect the relative risk of each of the considered risk factors. Only one of the recent studies made an attempt to consider the not uncommon scenario of coexistence of multiple stroke risk factors in a patient by providing some data on the cumulative risk vs. the risk of stand-alone risk factors. Optimal discrimination between risk strata, the absence of overlap of the respective CIs, was observed for 3 RSMs. Regarding the presented discriminative capability between RSMs, we must be aware that not all methods of calculating the c-statistic provide adequate discrimination levels, as well as that cross-study comparison being impaired by the differences in study populations. Finally, because of the heterogeneity of studies included in the systematic review, a meta-analysis of the data was not feasible.

\section{Conclusion}

The importance of stroke prevention in AF has lead to worldwide focus on this topic, resulting in an (ever) increasing body of evidence on stroke risk factors and the development and validation of stroke RSMs. Recognizing the importance of establishing individual profiles through risk factor-based assessment for both risk of stroke and major bleeding, and to develop an integrative ('net clinical benefit') approach, is pivotal to reducing adverse clinical events in patients with AF.

Perhaps the best option is the clinical practice shift that is strongly recommended in the new 2012 focussed update of the European Society of Cardiology guidelines on atrial fibrillation, which is towards more focus on identification of 'truly low-risk' patients with AF (that is, 'age $<65$ and lone AF (irrespective of gender) or $\mathrm{CHA}_{2} \mathrm{DS}_{2}-\mathrm{VASc}$ score $=0$ '), who do not need any antithrombotic therapy) instead of trying to focus on identifying 'high-risk' patients. ${ }^{92}$

\section{Acknowledgments}

Professor Lip provided the concept for the systematic review. The data were organized, interpreted and the manuscript wholly drafted/revised by the co-authors of this article, who take full responsibility for the article.

Assistance for the initial information searches was provided by Sue Libretto from Paraxel, funded by an educational grant from Boehringer Ingelheim. These were supplemented by additional independent searches from the authors.

No editorial support was provided nor funded.

\section{Disclosures}

Competing Interests: Professor Lip has served as a consultant for Bayer, Astellas, Merck, Sanofi, BMS/Pfizer, Daiichi-Sankyo, Biotronik, Portola and Boehringer Ingelheim and has been on the speakers bureau for Bayer, BMS/Pfizer, Boehringer Ingelheim, and Sanofi Aventis. 
Dr Lane has received research funding and/or honoraria for educational symposia from Bayer Healthcare, Boehringer Ingelheim, and Bristol-Myers-Squibb/Pfizer in relation to atrial fibrillation.

\section{References}

1. Heeringa J, van der Kuip DA, Hofman A, Kors JA, van Herpen G, Stricker BH, et al. Prevalence, incidence and lifetime risk of atrial fibrillation: The Rotterdam study. Eur Heart J 2006; 27: 949-953.

2. Benjamin EJ, Wolf PA, D'Agostino RB, Silbershatz H, Kannel WB, Levy D. Impact of atrial fibrillation on the risk of death: The Framingham Heart Study. Circulation 1998; 98: 946-952.

3. Hughes M, Lip GY. Stroke and thromboembolism in atrial fibrillation: A systematic review of stroke risk factors, risk stratification schema and cost effectiveness data. Thromb Haemost 2008; 99: 295 304.

4. Risk factors for stroke and efficacy of antithrombotic therapy in atrial fibrillation: Analysis of pooled data from five randomized controlled trials. Arch Intern Med 1994; 154: 1449-1457.

5. Hart RG, Pearce LA, Aguilar MI. Meta-analysis: Antithrombotic therapy to prevent stroke in patients who have nonvalvular atrial fibrillation. Ann Intern Med 2007; 146: 857-867.

6. Connolly SJ, Ezekowitz MD, Yusuf S, Eikelboom J, Oldgren J, Parekh $\mathrm{A}$, et al. Dabigatran versus warfarin in patients with atrial fibrillation. N Engl J Med 2009; 361: 1139-1151.

7. Granger CB, Alexander JH, McMurray JJ, Lopes RD, Hylek EM, Hanna M, et al. Apixaban versus warfarin in patients with atrial fibrillation. N Engl J Med 2011; 365: 981-992.

8. Patel MR, Mahaffey KW, Garg J, Pan G, Singer DE, Hacke W, et al. Rivaroxaban versus warfarin in nonvalvular atrial fibrillation. $N$ Engl J Med 2011; 365: 883-891.

9. Connolly SJ, Eikelboom J, Joyner C, Diener HC, Hart R, Golitsyn S, et al. Apixaban in patients with atrial fibrillation. N Engl J Med 2011; 364: $806-817$.

10. Mant J, Hobbs FD, Fletcher K, Roalfe A, Fitzmaurice D, Lip GY, et al. Warfarin versus aspirin for stroke prevention in an elderly community population with atrial fibrillation (the Birmingham Atrial Fibrillation Treatment of the Aged Study, BAFTA): A randomised controlled trial. Lancet 2007; 370: 493-503.

11. van Walraven C, Hart RG, Connolly S, Austin PC, Mant J, Hobbs FD, et al. Effect of age on stroke prevention therapy in patients with atrial fibrillation: The atrial fibrillation investigators. Stroke 2009; 40: 1410 1416.

12. Healey JS, Hart RG, Pogue J, Pfeffer MA, Hohnloser SH, De Caterina $\mathrm{R}$, et al. Risks and benefits of oral anticoagulation compared with clopidogrel plus aspirin in patients with atrial fibrillation according to stroke risk: The atrial fibrillation clopidogrel trial with irbesartan for prevention of vascular events (ACTIVE-W). Stroke 2008; 39: $1482-1486$.

13. Gorin L, Fauchier L, Nonin E, de Labriolle A, Haguenoer K, Cosnay $\mathrm{P}$, et al. Antithrombotic treatment and the risk of death and stroke in patients with atrial fibrillation and a CHADS2 score $=1$. Thromb Haemost 2010; 103: 833-840.

14. Gallagher AM, Setakis E, Plumb JM, Clemens A, van Staa TP. Risks of stroke and mortality associated with suboptimal anticoagulation in atrial fibrillation patients. Thromb Haemost 2011; 106: 968-977.

15. Lip GY, Nieuwlaat R, Pisters R, Lane DA, Crijns HJ. Refining clinical risk stratification for predicting stroke and thromboembolism in atrial fibrillation using a novel risk factor-based approach: The euro heart survey on atrial fibrillation. Chest 2010; 137: 263-272.

16. Olesen JB, Lip GY, Hansen ML, Hansen PR, Tolstrup JS, Lindhardsen $\mathrm{J}$, et al. Validation of risk stratification schemes for predicting stroke and thromboembolism in patients with atrial fibrillation: Nationwide cohort study. BMJ 2011; 342: d124.

17. Camm AJ, Kirchhof P, Lip GY, Schotten U, Savelieva I, Ernst S, et al. Guidelines for the management of atrial fibrillation: The Task Force for the Management of Atrial Fibrillation of the European Society of Cardiology (ESC). Europace 2010; 12: 1360-1420.

18. Cabin HS, Clubb KS, Hall C, Perlmutter RA, Feinstein AR. Risk for systemic embolization of atrial fibrillation without mitral stenosis. Am J Cardiol 1990; 65: 1112-1116.

19. Frost L, Vukelic Andersen L, Godtfredsen J, Mortensen LS. Age and risk of stroke in atrial fibrillation: Evidence for guidelines? Neuroepidemiology 2007; 28: 109-115.

20. Inoue $\mathrm{H}$, Atarashi $\mathrm{H}$. Risk factors for thromboembolism in patients with paroxysmal atrial fibrillation. Am J Cardiol 2000; 86: 852-855.

21. Lin LY, Lee CH, Yu CC, Tsai CT, Lai LP, Hwang JJ, et al. Risk factors and incidence of ischemic stroke in Taiwanese with nonvalvular atrial fibrillation: A nation wide database analysis. Atherosclerosis 2011; 217: 292-295.
22. Moulton AW, Singer DE, Haas JS. Risk factors for stroke in patients with nonrheumatic atrial fibrillation: A case-control study. Am J Med 1991; 91: 156-161.

23. Ruigomez A, Johansson S, Wallander MA, Edvardsson N, Garcia Rodriguez LA. Risk of cardiovascular and cerebrovascular events after atrial fibrillation diagnosis. Int J Cardiol 2009; 136: 186-192.

24. van Latum JC, Koudstaal PJ, Venables GS, van Gijn J, Kappelle LJ, Algra A. Predictors of major vascular events in patients with a transient ischemic attack or minor ischemic stroke and with nonrheumatic atrial fibrillation: European Atrial Fibrillation Trial (EAFT) Study Group. Stroke 1995; 26: 801-806.

25. Van Staa TP, Setakis E, Di Tanna GL, Lane DA, Lip GY. A comparison of risk stratification schemes for stroke in 79,884 atrial fibrillation patients in general practice. J Thromb Haemost 2011; 9: 3948.

26. The Stroke Prevention in Atrial Fibrillation Investigators. Predictors of thromboembolism in atrial fibrillation. II: Echocardiographic features of patients at risk. Ann Intern Med 1992; 116: 6-12.

27. The SPAF III Writing Committee for the Stroke Prevention in Atrial Fibrillation Investigators. Patients with nonvalvular atrial fibrillation at low risk of stroke during treatment with aspirin: Stroke Prevention in Atrial Fibrillation III Study. JAMA 1998; 279: 1273-1277.

28. Hart RG, Pearce LA, McBride R, Rothbart RM, Asinger RW; The Stroke Prevention in Atrial Fibrillation (SPAF) Investigators. Factors associated with ischemic stroke during aspirin therapy in atrial fibrillation: Analysis of 2012 participants in the SPAF I-III clinical trials. Stroke 1999; 30: 1223-1229.

29. Hart RG, Pearce LA, Rothbart RM, McAnulty JH, Asinger RW, Halperin JL; Stroke Prevention in Atrial Fibrillation Investigators. Stroke with intermittent atrial fibrillation: Incidence and predictors during aspirin therapy. J Am Coll Cardiol 2000; 35: 183-187.

30. Hobbs FD, Roalfe AK, Lip GY, Fletcher K, Fitzmaurice DA, Mant J. Performance of stroke risk scores in older people with atrial fibrillation not taking warfarin: Comparative cohort study from BAFTA trial. $B M J$ 2011; 342: d3653.

31. Nakagami H, Yamamoto K, Ikeda U, Mitsuhashi T, Goto T, Shimada $\mathrm{K}$. Mitral regurgitation reduces the risk of stroke in patients with nonrheumatic atrial fibrillation. Am Heart J 1998; 136: 528-532.

32. Petersen P, Kastrup J, Helweg-Larsen S, Boysen G, Godtfredsen J. Risk factors for thromboembolic complications in chronic atrial fibrillation: The Copenhagen AFASAK study. Arch Intern Med 1990; 150: $819-821$

33. Stollberger C, Chnupa P, Abzieher C, Langer T, Finsterer J, Klem I, et al. Mortality and rate of stroke or embolism in atrial fibrillation during long-term follow-up in the embolism in left atrial thrombi (ELAT) study. Clin Cardiol 2004; 27: 40-46.

34. Echocardiographic predictors of stroke in patients with atrial fibrillation: A prospective study of 1066 patients from 3 clinical trials. Arch Intern Med 1998; 158: 1316-1320.

35. Stollberger C, Chnupa P, Kronik G, Brainin M, Finsterer J, Schneider $\mathrm{B}$, et al. Transesophageal echocardiography to assess embolic risk in patients with atrial fibrillation: ELAT [Embolism in Left Atrial Thrombi] Study Group. Ann Intern Med 1998; 128: 630-638.

36. Wang TJ, Massaro JM, Levy D, Vasan RS, Wolf PA, D'Agostino RB, et al. A risk score for predicting stroke or death in individuals with new-onset atrial fibrillation in the community: The Framingham Heart Study. JAMA 2003; 290: 1049-1056.

37. Aronow WS, Gutstein H, Hsieh FY. Risk factors for thromboembolic stroke in elderly patients with chronic atrial fibrillation. Am J Cardiol 1989; 63: 366-367.

38. Aronow WS, Ahn C, Kronzon I, Gutstein H. Risk factors for new thromboembolic stroke in patients $>$ or $=62$ years of age with chronic atrial fibrillation. Am J Cardiol 1998; 82: 119-121.

39. Fang MC, Singer DE, Chang Y, Hylek EM, Henault LE, Jensvold NG, et al. Gender differences in the risk of ischemic stroke and peripheral embolism in atrial fibrillation: The AnTicoagulation and Risk factors In Atrial fibrillation (ATRIA) study. Circulation 2005; 112: $1687-1691$.

40. The Stroke Prevention in Atrial Fibrillation Investigators. Predictors of thromboembolism in atrial fibrillation. I: Clinical features of patients at risk. Ann Intern Med 1992; 116: 1-5.

41. The Stroke Prevention in Atrial Fibrillation Study. Risk factors for thromboembolism during aspirin therapy in patients with atrial fibrillation. J Stroke Cerebrovasc Dis 1995; 5: 147-157.

42. Seidl K, Hauer B, Schwick NG, Zellner D, Zahn R, Senges J. Risk of thromboembolic events in patients with atrial flutter. Am J Cardiol 1998; 82: 580-583.

43. Go AS, Hylek EM, Chang Y, Phillips KA, Henault LE, Capra AM, et al. Anticoagulation therapy for stroke prevention in atrial fibrillation: How well do randomized trials translate into clinical practice? 
JAMA 2003; 290: 2685-2692.

44. Rasmussen LH, Larsen TB, Due KM, Tjonneland A, Overvad K, Lip GY. Impact of vascular disease in predicting stroke and death in patients with atrial fibrillation: The Danish Diet, Cancer and Health cohort study. J Thromb Haemost 2011; 9: 1301-1307.

45. Heeringa J, Conway DS, van der Kuip DA, Hofman A, Breteler MM, Lip GY, et al. A longitudinal population-based study of prothrombotic factors in elderly subjects with atrial fibrillation: The Rotterdam Study 1990-1999. J Thromb Haemost 2006; 4: 1944-1949.

46. Friberg L, Hammar N, Rosenqvist M. Stroke in paroxysmal atrial fibrillation: Report from the Stockholm Cohort of Atrial Fibrillation. Eur Heart J 2010; 31: 967-975.

47. Fuster V, Ryden LE, Asinger RW, Cannom DS, Crijns HJ, Frye RL, et al. ACC/AHA/ESC guidelines for the management of patients with atrial fibrillation: A report of the American College of Cardiology/ American Heart Association Task Force on Practice Guidelines and the European Society of Cardiology Committee for Practice Guidelines and Policy Conferences (Committee to develop guidelines for the management of patients with atrial fibrillation) developed in collaboration with the North American Society of Pacing and Electrophysiology. Eur Heart J 2001; 22: 1852-1923.

48. Hirsh J, Dalen J, Guyatt G. The sixth (2000) ACCP guidelines for antithrombotic therapy for prevention and treatment of thrombosis. American College of Chest Physicians. Chest 2001; 119(Suppl): $1 \mathrm{~S}-2 \mathrm{~S}$

49. Gage BF, Waterman AD, Shannon W, Boechler M, Rich MW, Radford MJ. Validation of clinical classification schemes for predicting stroke: Results from the National Registry of Atrial Fibrillation. JAMA 2001; 285: $2864-2870$

50. Pearce LA, Hart RG, Halperin JL. Assessment of three schemes for stratifying stroke risk in patients with nonvalvular atrial fibrillation. Am J Med 2000; 109: 45-51

51. van Walraven C, Hart RG, Wells GA, Petersen P, Koudstaal PJ, Gullov AL, et al. A clinical prediction rule to identify patients with atrial fibrillation and a low risk for stroke while taking aspirin. Arch Intern Med 2003; 163: 936-943.

52. Gage BF, van Walraven C, Pearce L, Hart RG, Koudstaal PJ, Boode BS, et al. Selecting patients with atrial fibrillation for anticoagulation: Stroke risk stratification in patients taking aspirin. Circulation 2004; 110: 2287-2292.

53. Caro JJ, O'Brien JA, Klittich WS, Jackson JD. The economic impact of warfarin prophylaxis in nonvalvular atrial fibrillation. Dis Management Clin Outcomes 1997; 1: 54-60.

54. Eckman MH, Levine HJ, Salem DN, Pauker SG. Making decisions about antithrombotic therapy in heart disease: Decision analytic and cost-effectiveness issues. Chest 1998; 114(Suppl): 699S-714S

55. Gage BF, Cardinalli AB, Albers GW, Owens DK. Cost-effectiveness of warfarin and aspirin for prophylaxis of stroke in patients with nonvalvular atrial fibrillation. JAMA 1995; 274: 1839-1845.

56. Lightowlers S, McGuire A. Cost-effectiveness of anticoagulation in nonrheumatic atrial fibrillation in the primary prevention of ischemic stroke. Stroke 1998; 29: 1827-1832.

57. Desbiens NA. Deciding on anticoagulating the oldest old with atrial fibrillation: Insights from cost-effectiveness analysis. $J$ Am Geriatr Soc 2002; 50: 863-869.

58. Gustafsson C, Asplund K, Britton M, Norrving B, Olsson B, Marke LA. Cost effectiveness of primary stroke prevention in atrial fibrillation: Swedish national perspective. BMJ 1992; 305: 1457-1460.

59. Sorensen SV, Dewilde S, Singer DE, Goldhaber SZ, Monz BU, Plumb JM. Cost-effectiveness of warfarin: Trial versus "real-world" stroke prevention in atrial fibrillation. Am Heart J 2009; 157: 1064-1073.

60. Thomson R, Parkin D, Eccles M, Sudlow M, Robinson A. Decision analysis and guidelines for anticoagulant therapy to prevent stroke in patients with atrial fibrillation. Lancet 2000; 355: 956-962.

61. Mercaldi CJ, Ciarametaro M, Hahn B, Chalissery G, Reynolds MW, Sander SD, et al. Cost efficiency of anticoagulation with warfarin to prevent stroke in medicare beneficiaries with nonvalvular atrial fibrillation. Stroke 2011; 42: 112-118

62. Jowett S, Bryan S, Mant J, Fletcher K, Roalfe A, Fitzmaurice D, et al Cost effectiveness of warfarin versus aspirin in patients older than 75 years with atrial fibrillation. Stroke 2011; 42: 1717-1721.

63. Freeman JV, Zhu RP, Owens DK, Garber AM, Hutton DW, Go AS, et al. Cost-effectiveness of dabigatran compared with warfarin for stroke prevention in atrial fibrillation. Ann Intern Med 2011; 154: $1-11$.

64. Shah SV, Gage BF. Cost-effectiveness of dabigatran for stroke prophylaxis in atrial fibrillation. Circulation 2011; 123: 2562-2570.

65. Sorensen SV, Kansal AR, Connolly S, Peng S, Linnehan J, BradleyKennedy C, et al. Cost-effectiveness of dabigatran etexilate for the prevention of stroke and systemic embolism in atrial fibrillation: A
Canadian payer perspective. Thromb Haemost 2011; 105: 908-919.

66. Banerjee A, Lane DA, Torp-Pedersen C, Lip GY. Net clinical benefit of new oral anticoagulants (dabigatran, rivaroxaban, apixaban) versus no treatment in a 'real world' atrial fibrillation population: A modelling analysis based on a nationwide cohort study. Thromb Haemost 2012; 107: 584-589.

67. Andersen LV, Mortensen LS, Lip GY, Lindholt JS, Faergeman O, Henneberg EW, et al. Atrial fibrillation and upper limb thromboembolectomy: A national cohort study. J Thromb Haemost 2011; 9: $1738-1743$.

68. Friberg L, Benson L, Rosenqvist M, Lip GY. Assessment of female sex as a risk factor in atrial fibrillation in Sweden: Nationwide retrospective cohort study. BMJ 2012; 344: e 3522 .

69. Olesen JB, Fauchier L, Lane DA, Taillandier S, Lip GY. Risk factors for stroke and thromboembolism in relation to age among patients with atrial fibrillation: The Loire Valley Atrial Fibrillation Project. Chest 2012; 141: 147-153.

70. Olesen JB, Lip GY, Lane DA, Køber L, Hansen ML, Karasoy D, et al. Vascular disease and stroke risk in atrial fibrillation: A nationwide cohort study. Am J Med 2012; 125: 826.e13-e23.

71. Potpara TS, Polovina MM, Licina MM, Marinkovic JM, Prostran MS, Lip GY. Reliable identification of "truly low" thromboembolic risk in patients initially diagnosed with "lone" atrial fibrillation: The Belgrade atrial fibrillation study. Circ Arrhythm Electrophysiol 2012; 5: $319-326$

72. Avgil Tsadok M, Jackevicius CA, Rahme E, Humphries KH, Behlouli $\mathrm{H}$, Pilote L. Sex differences in stroke risk among older patients with recently diagnosed atrial fibrillation. JAMA 2012; 307: 1952-1958

73. Dinh T, Baur LH, Pisters R, Kamp O, Verheugt FW, Smeets JL, et al Feasibility of TEE-guided stroke risk assessment in atrial fibrillationbackground, aims, design and baseline data of the TIARA pilot study. Neth Heart J 2011; 19: 214-222.

74. Kirchhof P, Lip GY, Van Gelder IC, Bax J, Hylek E, Kaab S, et al. Comprehensive risk reduction in patients with atrial fibrillation: Emerging diagnostic and therapeutic options: A report from the 3rd Atria Fibrillation Competence NETwork/European Heart Rhythm Association consensus conference. Europace 2012; 14: 8-27.

75. Lee S, Anglade MW, Pham D, Pisacane R, Kluger J, Coleman CI. Cost-effectiveness of rivaroxaban compared to warfarin for stroke prevention in atrial fibrillation. Am J Cardiol 2012 May 29 [Epub ahead of print].

76. Lee S, Anglade MW, Meng J, Hagstrom K, Kluger J, Coleman CI. Cost-effectiveness of apixaban compared with aspirin for stroke prevention in atrial fibrillation among patients unsuitable for warfarin. Circ Cardiovasc Qual Outcomes 2012; 5: 472-479.

77. Kansal AR, Sorensen SV, Gani R, Robinson P, Pan F, Plumb JM, et al. Cost-effectiveness of dabigatran etexilate for the prevention of stroke and systemic embolism in UK patients with atrial fibrillation. Heart 2012; 98: $573-578$.

78. Langkilde LK, Bergholdt Asmussen M, Overgaard M. Cost-effectiveness of dabigatran etexilate for stroke prevention in non-valvular atrial fibrillation: Applying RE-LY to clinical practice in Denmark. J Med Econ 2012; 15: 695-703.

79. Pink J, Lane S, Pirmohamed M, Hughes DA. Dabigatran etexilate versus warfarin in management of non-valvular atrial fibrillation in UK context: Quantitative benefit-harm and economic analyses. BMJ 2011; 343: d6333.

80. Pisters R, Lane DA, Nieuwlaat R, de Vos CB, Crijns HJ, Lip GY. A novel user-friendly score (HAS-BLED) to assess 1-year risk of major bleeding in patients with atrial fibrillation: The Euro Heart Survey. Chest 2010; 138: 1093-1100.

81. Hughes M, Lip GY. Risk factors for anticoagulation-related bleeding complications in patients with atrial fibrillation: A systematic review. Q J Med 2007; 100: 599-607.

82. Lip GY, Frison L, Halperin JL, Lane DA. Comparative validation of a novel risk score for predicting bleeding risk in anticoagulated patients with atrial fibrillation: The HAS-BLED (Hypertension, Abnormal Renal/Liver Function, Stroke, Bleeding History or Predisposition, Labile INR, Elderly, Drugs/Alcohol Concomitantly) score. J Am Coll Cardiol 2011; 57: 173-180.

83. Roldán V, Marín F, Fernández H, Manzano-Fernandez S, Gallego P, Valdés M, et al. Predictive value of the HAS-BLED and ATRIA bleeding scores for the risk of serious bleeding in a 'real world' anticoagulated atrial fibrillation population. Chest 2012 June 21 [Epub ahead of print]

84. Fang MC, Go AS, Chang Y, Borowsky LH, Pomernacki NK, Udaltsova $\mathrm{N}$, et al. A new risk scheme to predict warfarin-associated hemorrhage: The ATRIA (Anticoagulation and Risk Factors in Atrial Fibrillation) Study. J Am Coll Cardiol 2011; 58: 395-401.

85. Olesen JB, Lip GY, Lindhardsen J, Lane DA, Ahlehoff O, Hansen 
ML, et al. Risks of thromboembolism and bleeding with thromboprophylaxis in patients with atrial fibrillation: A net clinical benefit analysis using a 'real world' nationwide cohort study. Thromb Haemost 2011; 106: 739-749.

86. Go AS, Fang MC, Udaltsova N, Chang Y, Pomernacki NK, Borowsky $\mathrm{L}$, et al. Impact of proteinuria and glomerular filtration rate on risk of thromboembolism in atrial fibrillation: The anticoagulation and risk factors in atrial fibrillation (ATRIA) study. Circulation 2009; 119: $1363-1369$.

87. Singer DE, Albers GW, Dalen JE, Go AS, Halperin JL, Manning WJ. Antithrombotic therapy in atrial fibrillation: The Seventh ACCP Conference on Antithrombotic and Thrombolytic Therapy. Chest 2004; 126(Suppl): 429S-56S.

88. Lip GY, Lowe GD. ABC of atrial fibrillation: Antithrombotic treatment for atrial fibrillation. $B M J$ 1996; 312: 45-49.

89. Fuster V, Ryden LE, Cannom DS, Crijns HJ, Curtis AB, Ellenbogen KA, et al. ACC/AHA/ESC 2006 guidelines for the management of patients with atrial fibrillation-executive summary: A report of the American College of Cardiology/American Heart Association Task Force on Practice Guidelines and the European Society of Cardiology Committee for Practice Guidelines (Writing Committee to Revise the 2001 Guidelines for the Management of Patients with Atrial Fibrillation). Eur Heart J 2006; 27: 1979-2030.
90. Rietbrock S, Heeley E, Plumb J, van Staa T. Chronic atrial fibrillation: Incidence, prevalence, and prediction of stroke using the Congestive heart failure, Hypertension, Age $>75$, Diabetes mellitus, and prior Stroke or transient ischemic attack (CHADS2) risk stratification scheme. Am Heart J 2008; 156: 57-64.

91. Lip GY, Edwards SJ. Stroke prevention with aspirin, warfarin and ximelagatran in patients with non-valvular atrial fibrillation: A systematic review and meta-analysis. Thromb Res 2006; 118: 321-333.

92. Camm AJ, Lip GY, De Caterina R, Savelieva I, Atar D, Hohnloser SH, et al. 2012 focused update of the ESC Guidelines for the management of atrial fibrillation: An update of the 2010 ESC Guidelines for the management of atrial fibrillation * Developed with the special contribution of the European Heart Rhythm Association. Eur Heart J 2012 August 24 [Epub ahead of print] PubMed PMID: 22922413.

\section{Supplementary Files}

Supplementary File 1

Appendix S1.

Please find supplementary file(s);

http://dx.doi.org/10.1253/circj.CJ-12-1036 